\title{
Reverse Actuation of Polyelectrolyte Effect for in
}

\section{Vivo Antifouling}

Woojin Choi ${ }^{1}$, Sohyeon Park ${ }^{1}$, Jae-Sung Kwon ${ }^{2}$, Eun-Young Jang ${ }^{3}$, Ji-Yeong Kim ${ }^{4}$, Jiwoong

$\mathrm{Heo}^{1}$, YoungDeok Hwang ${ }^{5}$, Byeong-Su Kim ${ }^{6}$, Ji-Hoi Moon ${ }^{7}$, Sungwon Jung ${ }^{1}$, Sung-Hwan Choi ${ }^{*}$, Hwankyu Lee ${ }^{*, 8}$, Hyo-Won Ahn ${ }^{*}$, , Jinkee Hong ${ }^{*}, 1$

${ }^{1}$ Department of Chemical \& Biomolecular Engineering, College of Engineering, Yonsei University, 50 Yonsei-ro, Seodaemun-gu, Seoul, 03722, Republic of Korea

${ }^{2}$ Department and Research Institute of Dental Biomaterials and Bioengineering and BK21 FOUR Project, Yonsei University College of Dentistry, Seoul, 03722, Republic of Korea

${ }^{3}$ Department of Dentistry, Graduate School, Kyung Hee University, Seoul, 02447, Republic of Korea

${ }^{4}$ Department of Orthodontics, Institute of Craniofacial Deformity and BK21 FOUR Project, Yonsei University College of Dentistry, Seoul, 03722, Republic of Korea

${ }^{5}$ Paul H. Chook Department of Information Systems and Statistics, Baruch College CUNY, New York, NY, 10010, United States of America

${ }^{6}$ Department of Chemistry, Yonsei University, Seoul, 03722, Republic of Korea 
${ }^{7}$ Department of Oral Microbiology, School of Dentistry, Kyung Hee University, Seoul 02447, Republic of Korea

${ }^{8}$ Department of Chemical Engineering, Dankook University, Yongin-si, Gyeonggi-do, 16890, Republic of Korea

${ }^{9}$ Department of Orthodontics, School of Dentistry, Kyung Hee University, Seoul, 02447, Republic of Korea 
(a)

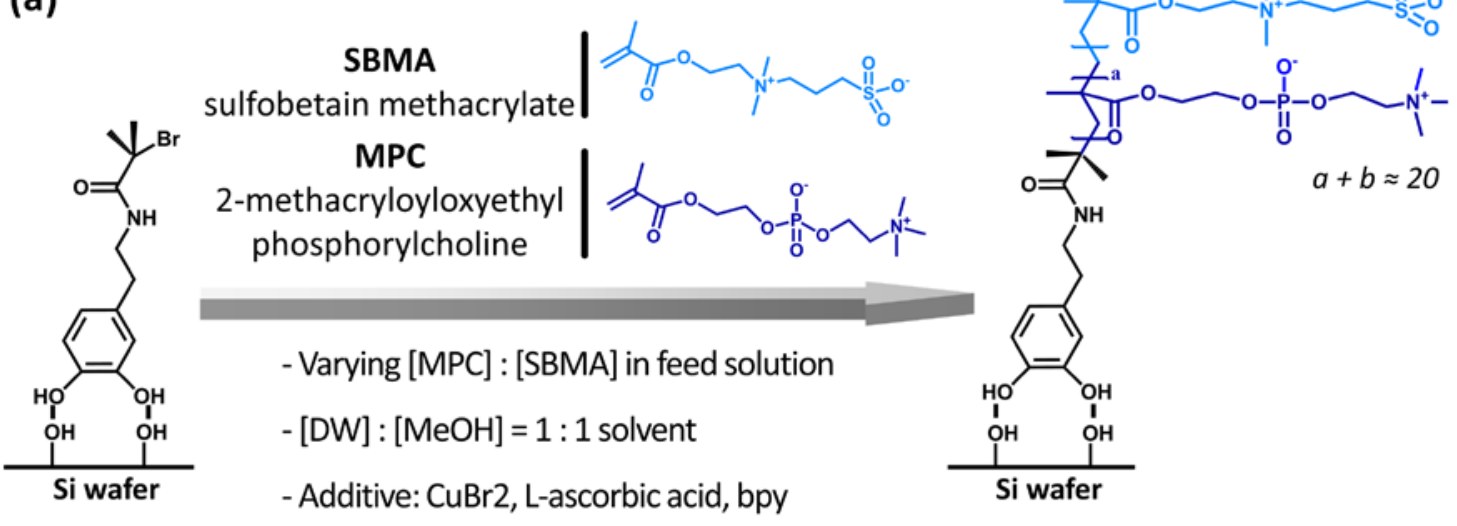

(b)
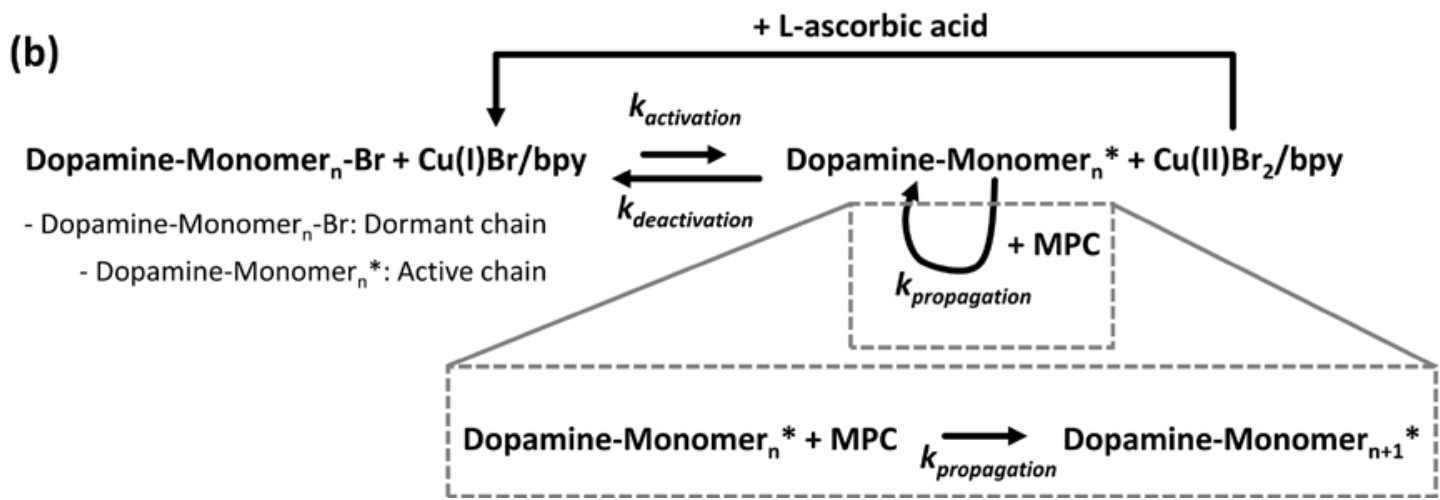

Figure S1. SI-ARGET ATRP reaction to synthesize ZPs (a) Brief schematic of the experimental procedure and (b) supposed reaction dynamics 

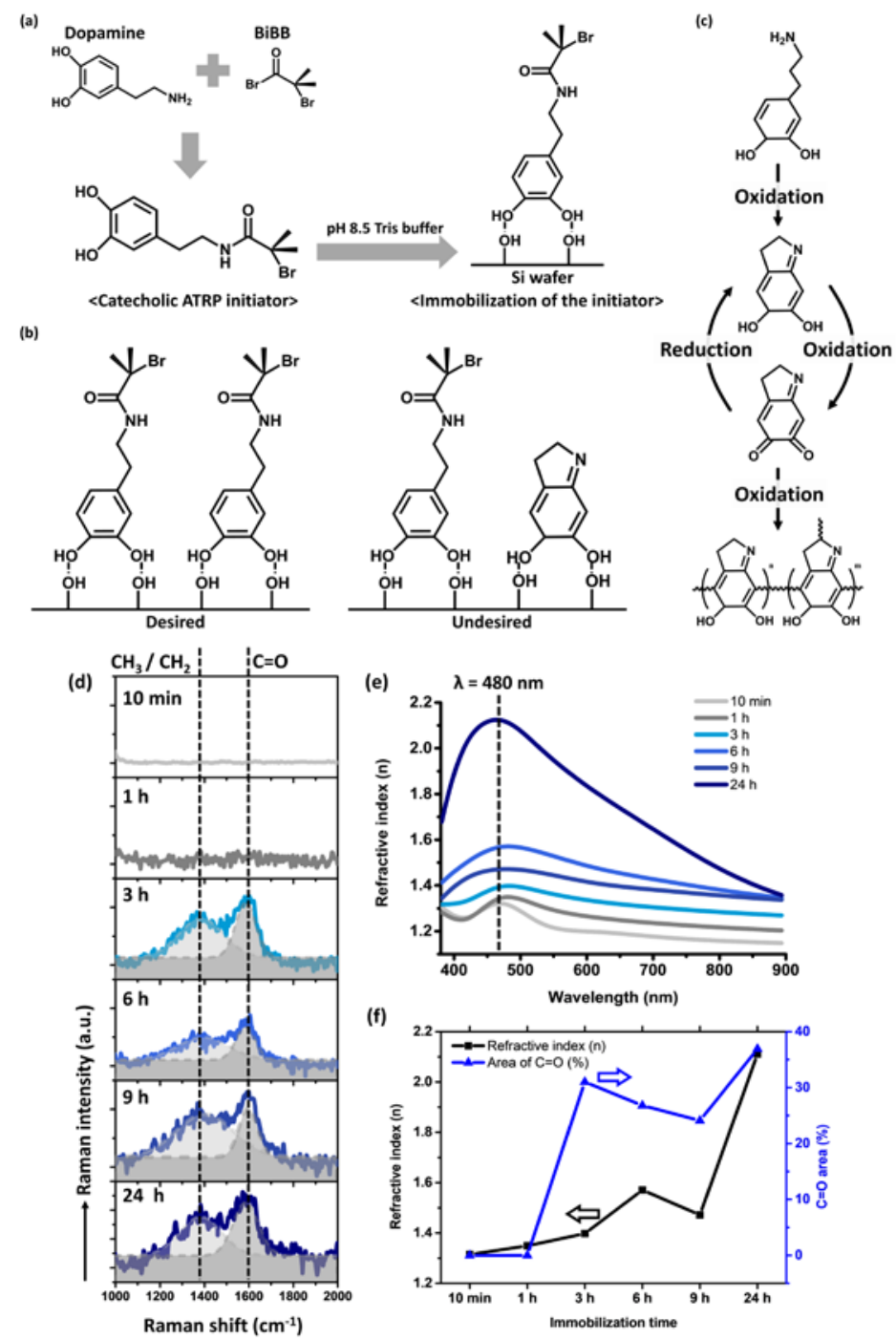

Figure S2. Optimization of initiator immobilization process (a) Synthetic and immobilization process of the catecholic ATRP initiator. (b) Chemical structures of possible conformations occurring in the immobilization step. The left represents the desired, and the right represents the undesired conformation. (c) Representative self-polymerization process of residual dopamine Optimizing the immobilization condition of catecholic ATRP initiators. (d) Raman spectrum, (e) refractive index in every immobilization time. (f) Summary of the two critical factors from Raman spectroscopy (blue) and ellipsometer (black). 

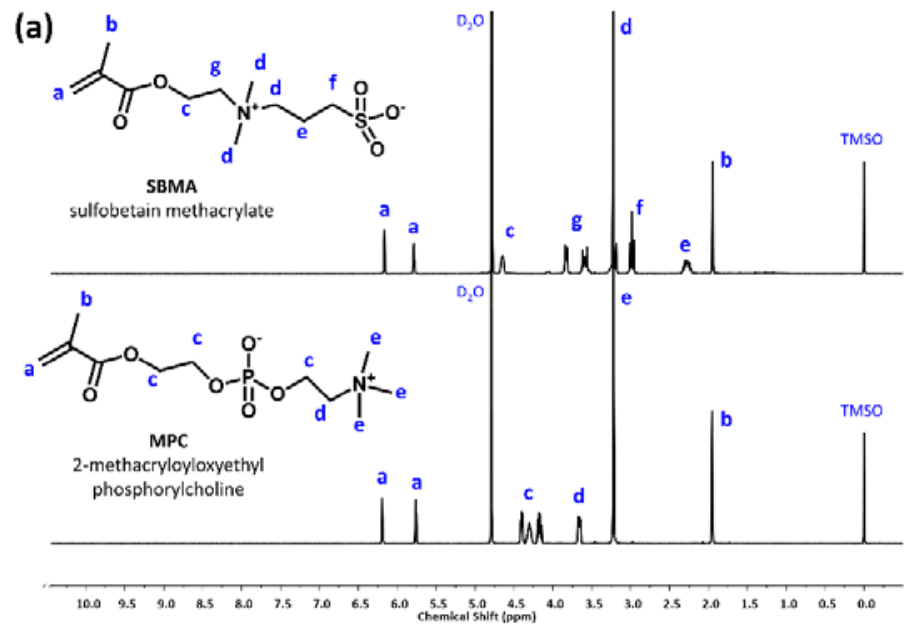

(b)

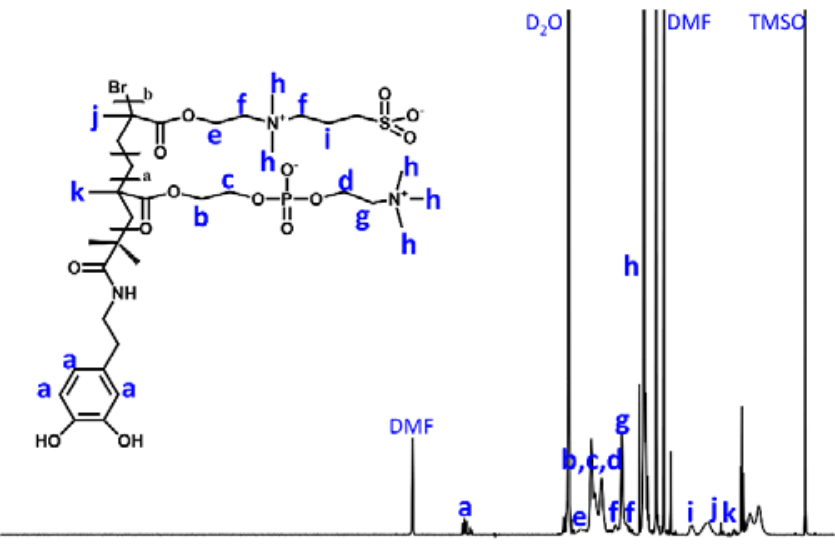

(c)

\begin{tabular}{cccc} 
* Polymer & In feed solution & \multicolumn{2}{c}{ In zwitterionic copolymer } \\
& ** [MPC]:[SBMA] & $M_{n}$ (g/mol) & ** [MPC]:[SBMA] \\
100MPC/OSBMA & $1.0: 0.0$ & 6,059 & $1.0: 0.0$ \\
80MPC/20SBMA & $0.8: 0.2$ & 5,688 & $0.83: 0.17$ \\
50MPC/50SBMA & $0.2: 0.8$ & 6,387 & $0.49: 0.51$
\end{tabular}

* $(X) \mathrm{MPC} /(Y)$ SBMA: $X, Y$ stood for the mole fraction within zwitterionic copolymer

** [MPC]:[SBMA] meant the mole fraction

Figure S3. Characterization of synthesized of ZPs (a) NMR spectrums of two commercial monomers. (Top) Sulfobetain methacrylate (SBMA) and (Bottom) 2-methacryloyloxyethyl phosphorylcholine (MPC). (b) Representative NMR spectrum of synthesized ZPs. (c) Characterization of the ZPs in terms of $M_{n}$ and mole fraction. 


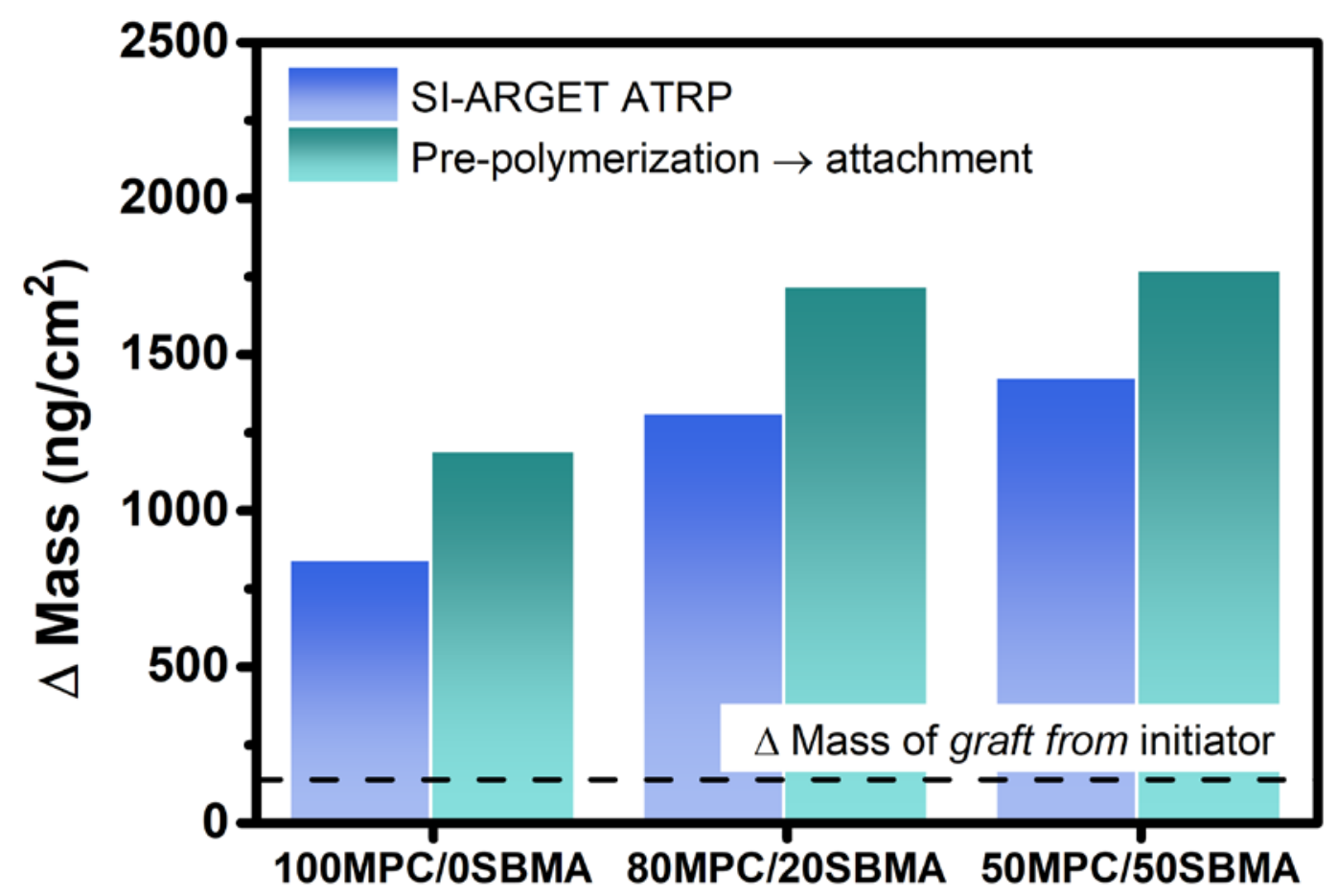

Figure S4. Surface coverage comparisons to double-check the measured molecular weight. The dashed line indicates the mass change after the immobilization of the catecholic ATRP initiator.

We designed the bypass strategy to measure the molecular weight of SI-ARGET ATRP ZPs, i.e., pre-polymerized ZPs within the identical condition of SI-ARGET ATRP were subjected to ${ }^{1} \mathrm{H}$ NMR. The ratio of monomers and initiator critically control the polymer's properties (e.g., molecular weight). Thus, we determined the attached initiators' mole based on the surface coverage (Dashed line in Figure S4). Then, the quantified amount of initiators was dissolved with zwitterionic monomers and additives for the pre-polymerization without substrate. The concentration of all reagents for pre-polymerization was identical with SI-ARGET ATRP process. The pre-polymerization has lasted $6 \mathrm{~h}$, the same duration as SI-ARGET ATRP. The obtained ZPs were subjected to ${ }^{1} \mathrm{H}$ NMR to evaluate the number-average molecular weight. For Figure S4, the pre-polymerized ZPs were spontaneously anchored to the QCM electrode (i.e., 'graft to’ approach) 
until the frequency changes saturated for $20 \mathrm{~min}$. The measured molecular weights (Figure S3c) were considered reasonable, concerning that the surface coverage difference between two approaches was insignificant (ca. 30\%).

It is practically challenging to accurately measure the in situ molecular weights of 'graft from' polymers. Thus, several studies have assumed that the molecular weights of "graft from" polymers might be similar to those of "graft to" polymers. ${ }^{1}$ Technically, the molecular weight may or may not be substantially different. ${ }^{2}$ Thus, the detachment of anchors polymers is ideal for measuring the accurate molecular weight. However, the detachment of catechol, the adhesive moiety of ZPs, ${ }^{3}$ was time-consuming since it required the external energy source (e.g, electrochemically oxidative potential ${ }^{4}$ or ultraviolet ${ }^{5}$ ). Notice that our objective was not focused on the detachment.

We also determined the surface coverage $(\Gamma)$, grafting density $(\sigma)$, and mean separation between polymer chains $(D)^{6,7}$, and these values are summarized in Table S1. In particular, the grafting density $(\sigma)$ is the average number of polymer chains per unit area $\left(\mathrm{nm}^{-2}\right)$ and is a widely used parameter. We regarded the optimized initiator immobilization process as the most influential factor increasing the grafting density (Figure S2). 
(a)

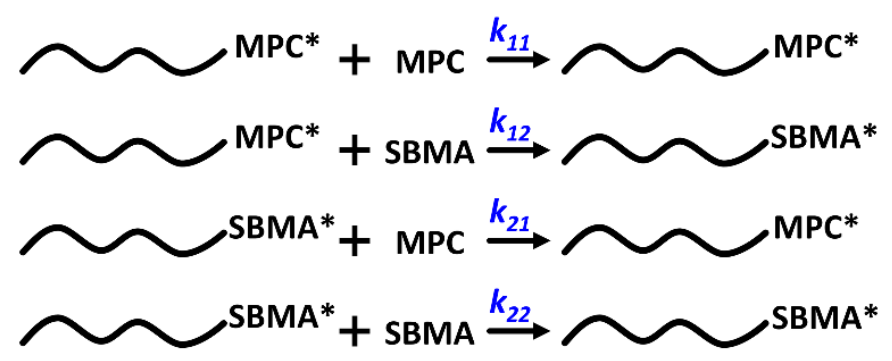

(b)

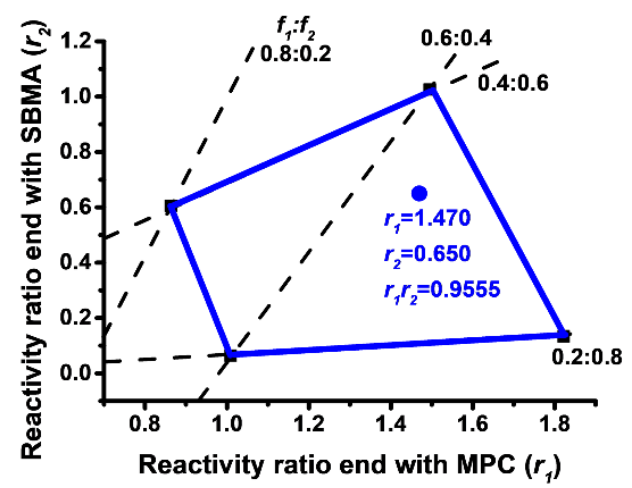

Figure S5. Conformation analysis of synthesized ZPs (a) Schematic demonstrating four kinds of growing chains end with MPC or SBMA. (b) Mayo-Lewis plot varying the mole fractions of monomers in the feed solution. The center of mass $\left(r_{1}, r_{2}\right)$ was calculated with the below equation.

Center of mass $\left(c_{x}, c_{y}\right)$

$$
\begin{aligned}
& c_{x}=\frac{1}{N A} \sum_{i=0}^{N-1}\left(x_{i}+x_{i+1}\right)\left(x_{i} y_{i+1}-x_{i+1} y_{i}\right) \\
& \text { and } c_{y}=\frac{1}{N A} \sum_{i=0}^{N-1}\left(y_{i}+y_{i+1}\right)\left(x_{i} y_{i+1}-x_{i+1} y_{i}\right) \\
& \text { where, Area }(A)=\frac{1}{2} \sum_{i=0}^{N-1}\left(x_{i} y_{i+1}-x_{i+1} y_{i}\right), \text { Number of points }(N)=4
\end{aligned}
$$

We analyzed the copolymerization dynamics and conformational type of copolymer using Mayo-Lewis plot (Equation (S1)). ${ }^{8} 9$

$$
r_{2}=\left(\frac{F_{2} f_{1}^{2}}{F_{1} f_{2}^{2}}\right) r_{1}+\frac{f_{1}}{f_{2}}\left(\frac{F_{2}}{F_{1}}-1\right) \cdots
$$

where arbitrary numbers 1 and 2 denote 2-methacryloyloxyethyl phosphorylcholine (MPC) and sulfobetaine methacrylate (SBMA), respectively, $f$ and $F$ are the monomer mole fractions in the feed solution and copolymer, respectively, and $r$ is the active chain reactivity end with 1 or 2 . Figure S5a shows the possible copolymerization kinetics. Because $r_{1}$ and $r_{2}$ are $k_{11} / k_{12}$ and $k_{22} / k_{21}$, 
the Mayo-Lewis plot (obtained using Equation (S1)) can model all kinds of copolymerization behavior. We quantified $r_{1}$ and $r_{2}$ by varying the mole fraction of the feed solution $(f)$ from 0 to 100 (Figure S5b). First, concerning the conformational type of copolymers, we concluded that each monomer was randomly distributed within the copolymer because $r_{1} r_{2}$ converged to 1.0 , as observed in other studies in which random copolymers were obtained by one-pot copolymerization. ${ }^{10}$ Crucially, a random copolymer is essential to achieve the anti-polyelectrolyte effect (APE) (see Equations (9) and (10) in the main document). Secondly, concerning copolymerization dynamics, we found that the active chain ends with MPC had higher reactivity than those of SBMA ( $r_{1}>r_{2}$; Figure S5b). The different affinities resulted in an unbalanced mole fraction between feed solution and random copolymer, especially in 50MPC/50SBMA (Figure S3c). 
(a)

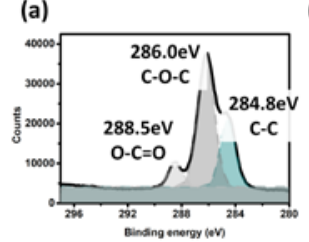

(b)

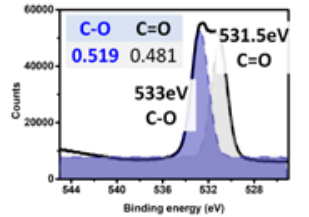

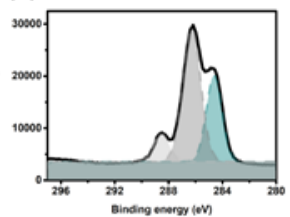

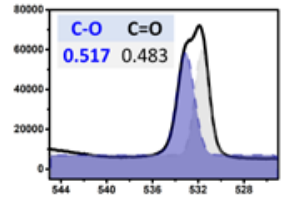

(c)
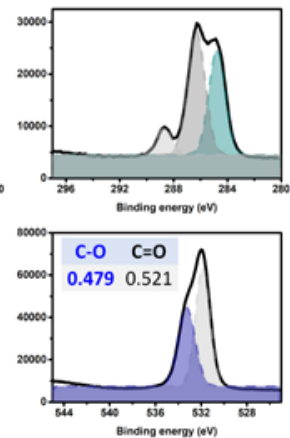

(d)

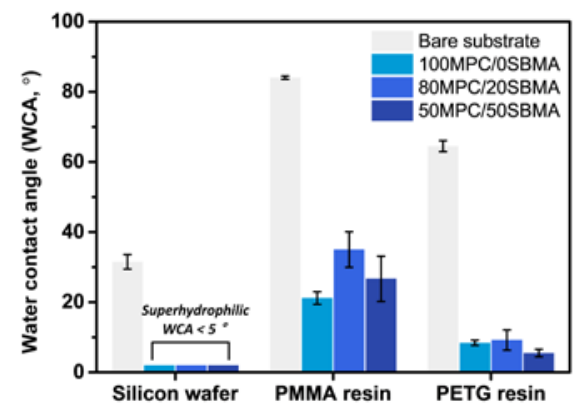

Figure S6. Characterization of synthesized of ZPs. The XPS spectrums at the $\mathrm{C}_{1 \mathrm{~s}}$ region $\left(1^{\text {st }}\right.$ row) and the $\mathrm{O}_{1 \mathrm{~s}}$ region ( ${ }^{\text {nd }}$ row) of (a) 100MPC/0SBMA, (b) 80MPC/20SBMA, and (c) 50MPC/50SBMA. The table inserted in the $\mathrm{O}_{1 \mathrm{~s}}$ spectrum indicates the percentage of normalized peak areas. (d) The water contact angle of $5 \mu \mathrm{L}$ droplet. The zwitterionic polymers were grafted from the three kinds of the substrate; Silicon wafer and commercial poly(methyl methacrylate)(PMMA), glycol-modified polyethylene terephthalate (PETG) resins for the dental clinic. 

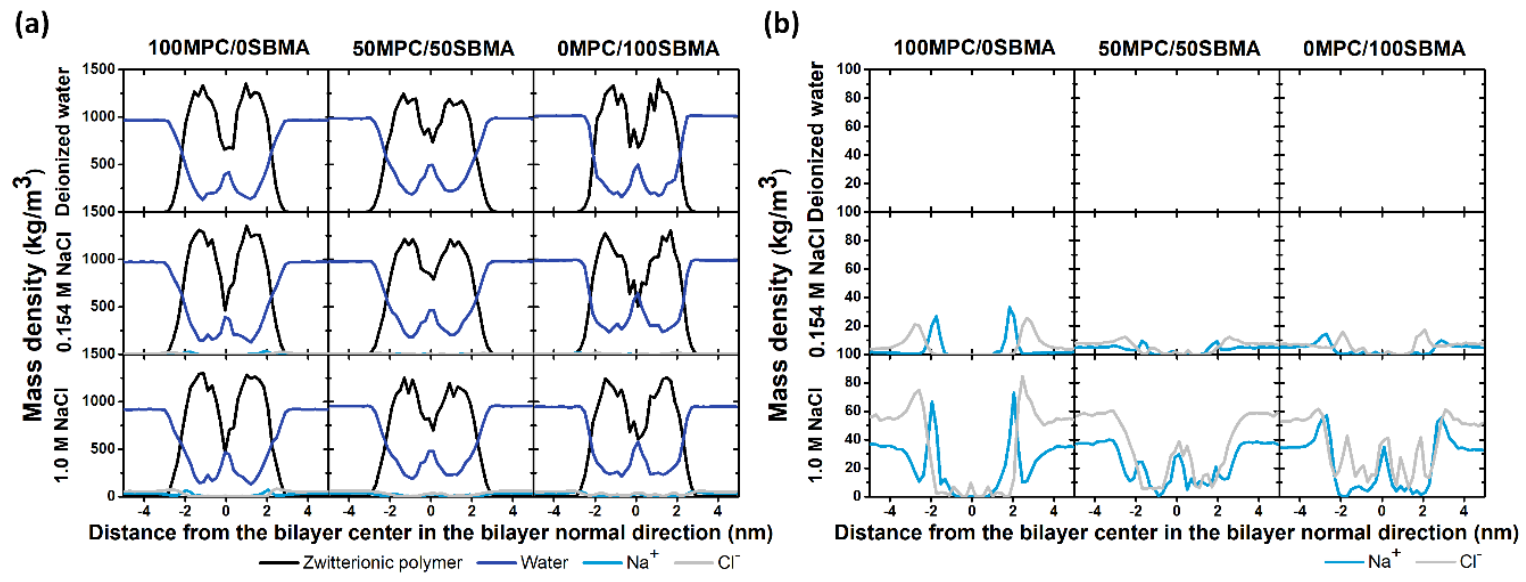

Figure S7. Mass density profiles of (a) zwitterionic polymers, water, and ions and (b) profiles highlighting only ions. 
Factor 1. Ion distribution

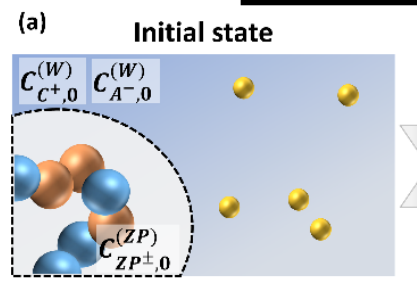

(b)

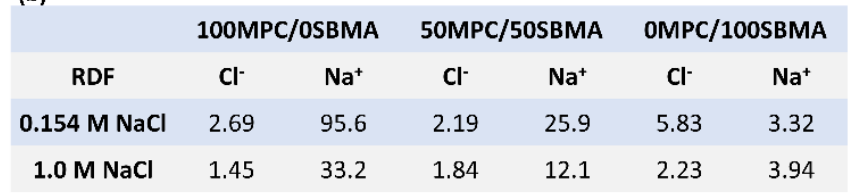

Factor 2. Charge screening

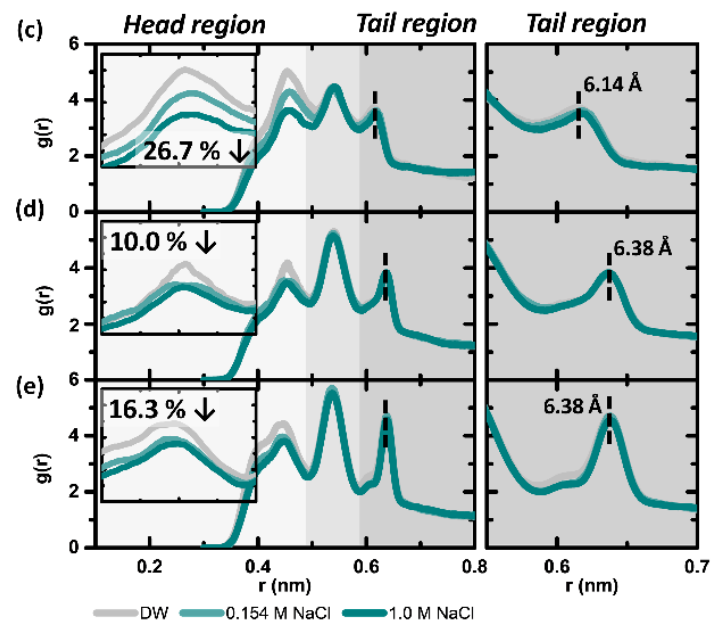

Figure S8. Detailed descriptions of molecular dynamics simulations. (a) The designed osmotic system referred to Georgiev et al. Cationic, and anionic moieties were presented as red and blue spheres, respectively, while yellow indicated ions. The thermodynamic factors are shown in the typical form $\alpha_{\gamma, \delta}^{(\beta)}$ where, $\alpha$ is the concentration $(C)$ or volume $(V), \beta$ is the phase of bulk water $(W)$ or zwitterionic polymer (ZP), $\gamma^{\square}$ is the cation $\left(C^{+}\right.$or $\left.Z P^{+}\right)$or anion $\left(A^{-}\right.$or $\left.Z P^{-}\right)$, and $\delta$ is the initial (0) or equilibrium state (e). For example, $C_{C^{+}, 0}^{(W)}$ means the concentration of the cation $C^{+}$in the initial water phase. Further, $C_{S, 0}^{(W)}$ denotes the concentration of added ions at the initial moment. (b) Summary of RDFs in Figure 3b for ions and polymers. Overall RDFs between anionic moieties and cationic moieties within polymers; (c) 100MPC/0SBMA, (d) 50MPC/50SBMA, and (e) 0MPC/100SBMA 
(a)

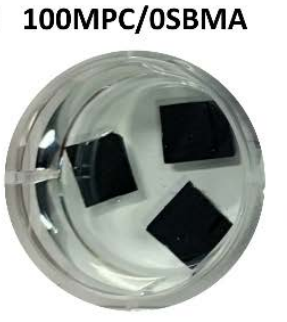

8OMPC/20SBMA

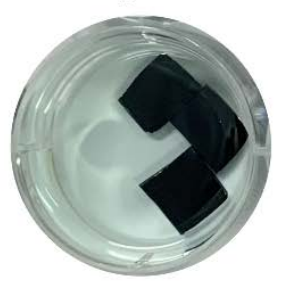

50MPC/50SBMA

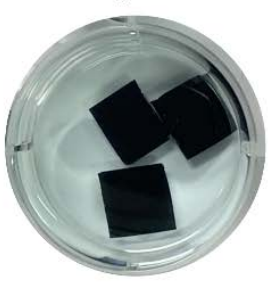

OMPC/100SBMA
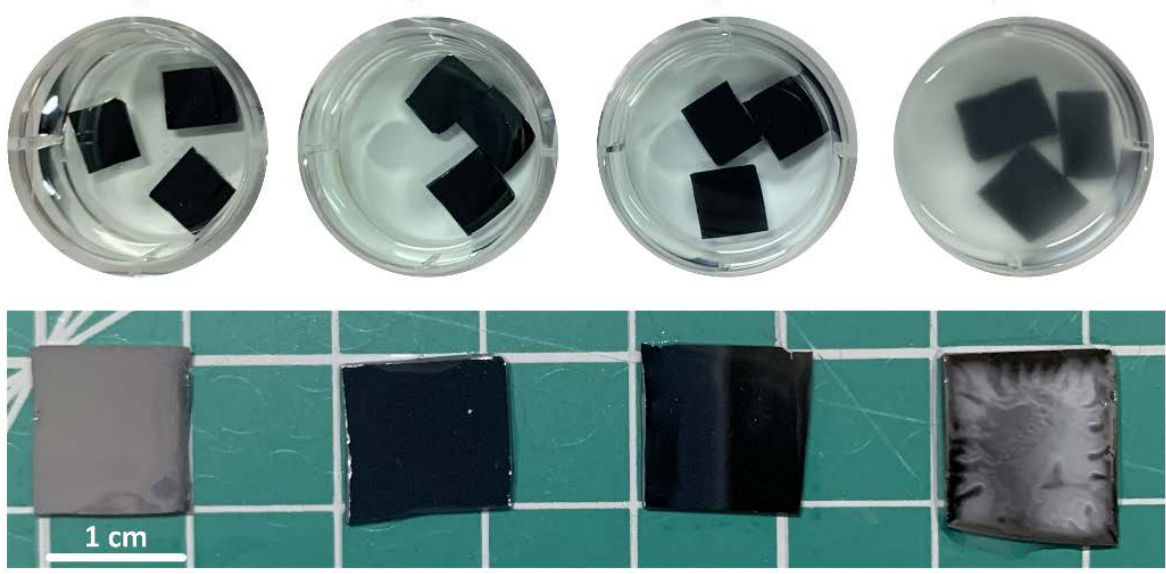

(b)
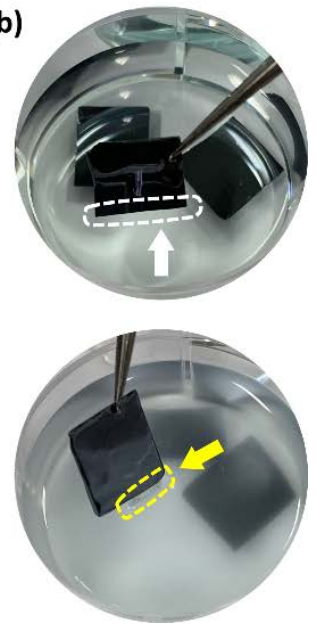

Figure S9. Photographs after the SI-ARGET ATRP. (a) The first row showed the photograph as soon as the SI-ARGET ATRP was finished. The residual catalyst copper-induced the light blue color, and the gel-like structure of 0MPC/100SBMA resulted in the opaque solution. The second row showed the silicon wafers’ photograph with zwitterionic polymers without further processing, such as washing or drying solvent. (b) Picture while taking out the silicon wafer after the polymerization. The white arrow indicated the non-sticky solution of 50MPC/50SBMA, while the yellow indicated a highly viscous solution of 0SBMA/100MPC. 


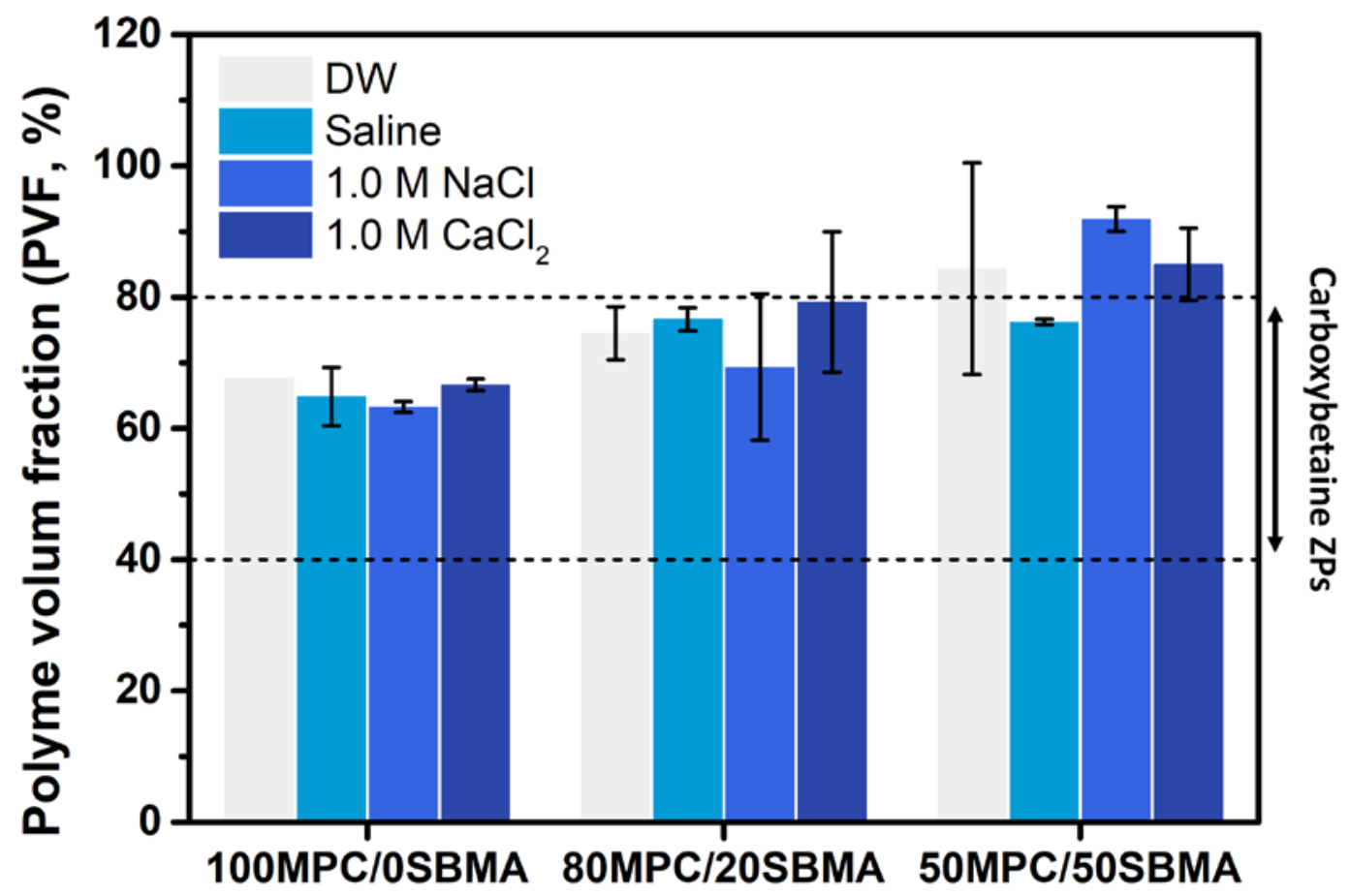

Figure S10. Polymer volume fraction (PVF) (unit: \%) of synthesized ZPs. PVFs were calculated using the below equation.

$$
\boldsymbol{P V} \boldsymbol{F}=\frac{\left(n_{p}^{2}-n_{b}^{2}\right)\left(n_{p-d r y}^{2}+2 n_{b}^{2}\right)}{\left(n_{p}^{2}+2 n_{b}^{2}\right)\left(n_{p-d r y}^{2}-n_{b}^{2}\right)}
$$

where, $n_{p}, n_{b}$, and $n_{p-d r y}$ is the refractive index of wet polymer brushes, media, and dry polymer brushes, respectively. 

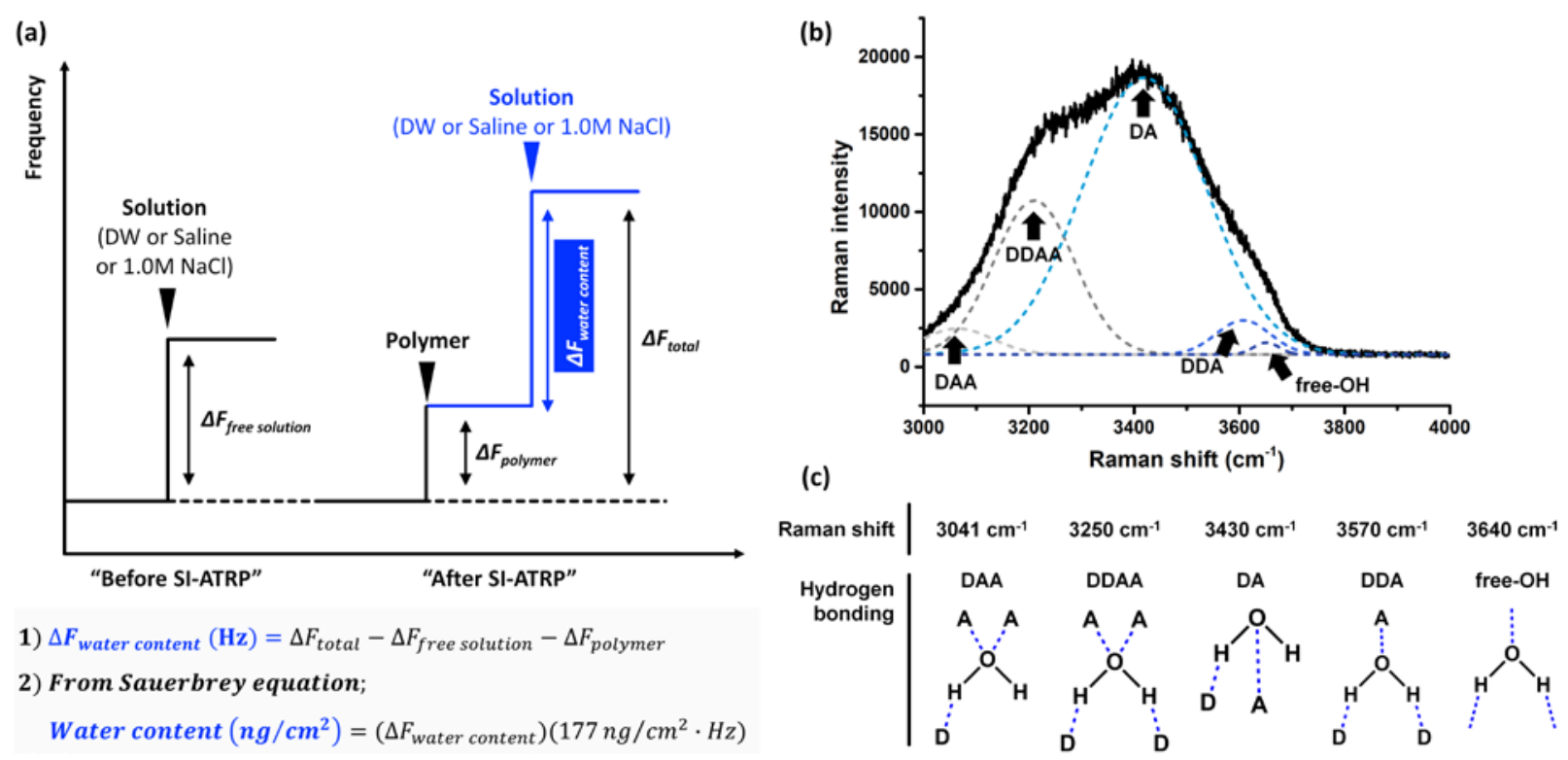

Figure S11. Methods to quantify the hydration efficiency. (a) Schematic illustration of in situ QCM measurement. Here, $\Delta F$ is the change in frequency $(\mathrm{Hz})$. (b) Five kinds of hydrogen bonding between zwitterionic moieties and water molecules. Mainly, $D$ represents the donor, and $A$ means the acceptor. (c) Representative Raman spectrum measured with 50MPC/50SBMA. The dashed lines represent five kinds of hydrogen bonding via Gaussian model deconvolution.

Raman spectroscopy is one of the optimal choice for visualizing the hydrogen bonding structure of water molecules. ${ }^{11} 12$ The vibrational spectroscopy (both FT-IR and Raman spectroscopy) is the reliable instrument to detect the local dynamics of water molecules (e.g., hydrogen-bonded structure). In particular, the collective oscillations of symmetric $\left(v_{1}\right)$, asymmetric $\left(v_{3}\right)$ stretching resulted in the shoulder peak range of $3000-4000 \mathrm{~cm}^{-1}$. The surrounding $-\mathrm{OH}$ groups delocalize one quantum of excitation, e.g., Fermi resonance of symmetric bending $\left(v_{2}\right)$ toward $v_{1}, v_{3}$ stretching. ${ }^{13,14}$ The resolution of spectrometers to classify such collective motions was sufficient for Raman spectroscopy. FT-IR is based on absorption, so it absorbs all similar vibrations. Otherwise, Raman spectroscopy is based on the Raman scattering. In detail, the instruments sensitively detect the shift of Stokes scattering versus Rayleigh scattering. Thus, Raman 
spectroscopy's sensitivity is appropriate to detect the water molecular vibrations since it is based on the relative shift, not absolute absorption (i.e., FT-IR).

In addition, since the Raman scattering occurs on a shorter timescale ( $\left.c a .10^{-14} \mathrm{~s}\right)$ than the relaxation of the $v_{1}$ vibration within water molecules $\left(\mathrm{ca} \cdot 10^{-11} \mathrm{~s}\right)$, structural changes to water molecules can be evaluated. ${ }^{11}$ In contrast, tools such as differential scanning calorimetry (10 s), X-ray diffraction (10 s), NMR $\left(10^{-6} \sim 10^{-11} \mathrm{~s}\right)$ hardly measure the changes of water at the molecular level. 


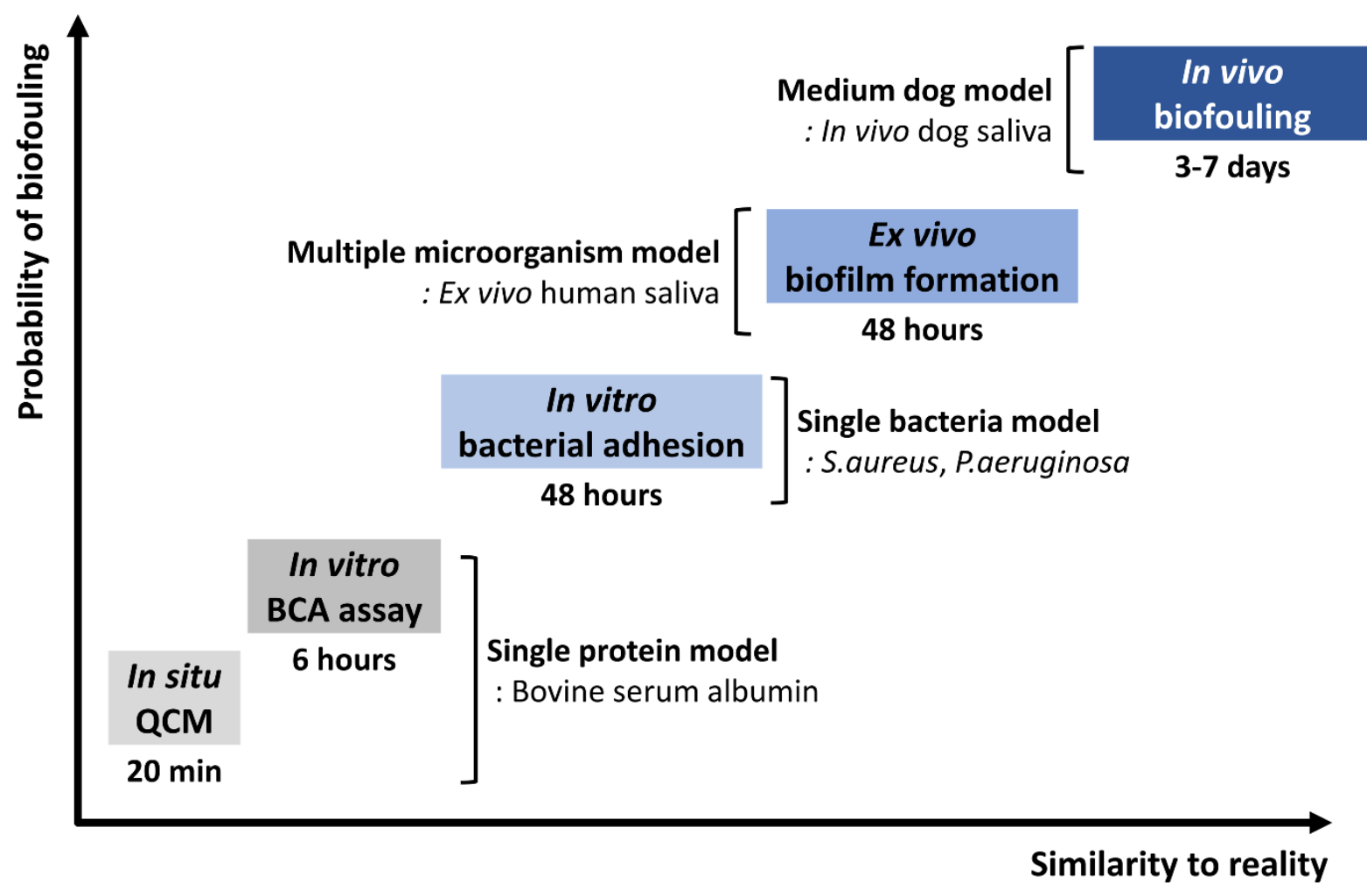

Figure S12. A diagram to describe the change in the experimental conditions, in which both possibilities of biofouling and realism would increase. 

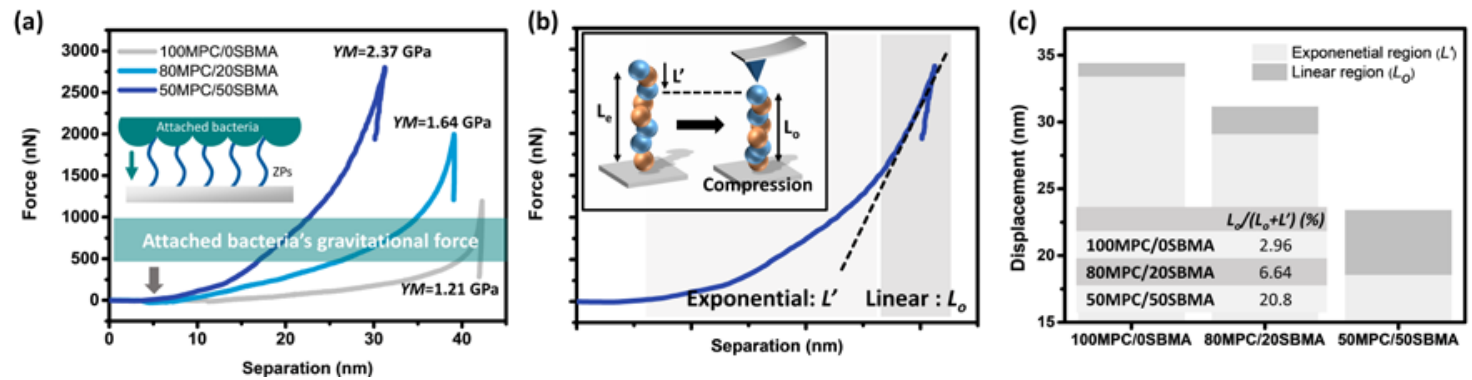

Figure S13. Nanomechanics of the zwitterionic polymers. (a) AFM nanoindentation to mimic in vitro bacterial adhesion. YM denotes Young's modulus. (b) Force-displacement curve of 50MPC/50SBMA. The inserted image represents the compression process. (c) The length of the exponential or linear region of ZPs. We determined the linear region by measuring the coefficient of determination (R2). R2 was 0.974 for 100MPC/0SBMA, 0.987 for 80MPC/20SBMA, and 0.994 for 50MPC/50SBMA. The inset table of Figure S13c suggested an index for the stiffness.

Antifouling performance is not expected if the ZPs are compressed by the gravitational force exerted by the attached bacteria, which would result in a loss of function. Thus, we investigated the nanomechanics of the ZPs via atomic force microscopy (AFM) nanoindentation to deduce the conformational changes induced by the bacterial gravitational force.

Typically, the mass of a single bacterium is approximately $1.0 \mathrm{pg}$, and in vitro seeding density was approximately $1.0 \times 10^{9}$ cells $\mathrm{mL}^{-1}$ (optical density $\approx 0.3$ at $600 \mathrm{~nm}$ ). In addition, since the ZPs imparted the hydrophilicity to the substrate, we assumed the parallel microcolony formation rather than vertical adhesion. ${ }^{15}$ Thus, we estimated an approximate force of 500-1000 nN applied to the ZPs after in vitro bacterial adhesion without any further proliferation. The forcedisplacement curves during nanoindentation of 100MPC/0SBMA, 80MPC/20SBMA, and 50MPC/50SBMA are shown in Figure S13a. 
When the AFM tip approached the ZPs, the force increased exponentially because of the repulsive steric hindrance of the tethered ZPs ${ }^{16}$. As shown in Figure S13b, the length of the exponential region is defined as $L^{\prime}$. The force further increases linearly because the ZP brush cannot be compressed any further by the external load. Thus, the length of the linear region $\left(L_{o}\right)$ gives the stiffness of the ZPs ${ }^{16}$. We concluded that the stiffness decreased in the following order: 50MPC/50SBMA > 80MPC/20SBMA > 100MPC/0SBMA (Figure S13c). In addition, the Young's modulus, another index of the stiffness, decreased in the same order: 50MPC/50SBMA $(2.37 \mathrm{GPa})>$ 80MPC/20SBMA (1.64 GPa) > 100MPC/0SBMA (1.21 GPa) (Figure S13a). We hypothesize that the self-crosslinking of SBMA resulted in the enhanced stiffness of 80MPC/20SBMA and 50MPC/50SBMA. Regardless of the stiffness, the threshold displacement to reach ca. $1000 \mathrm{nN}$ was considerable for all ZPs (Figure S13a).

Thus, all polymers might suffer from the compression and loss of functions due to the gravitational force of attached bacteria. However, note that AFM nanomechanical measurements were performed under dry conditions where the smart ZPs do not exhibit the APE. Figures 5-8 shows that 50MPC/50SBMA showed impressive antifouling performance under physiological conditions, in which 50MPC/50SBMA spontaneously exhibit APE. Thus, it was reasonable to conclude that APE had a significant role in the antifouling property of 50MPC/50SBMA. 

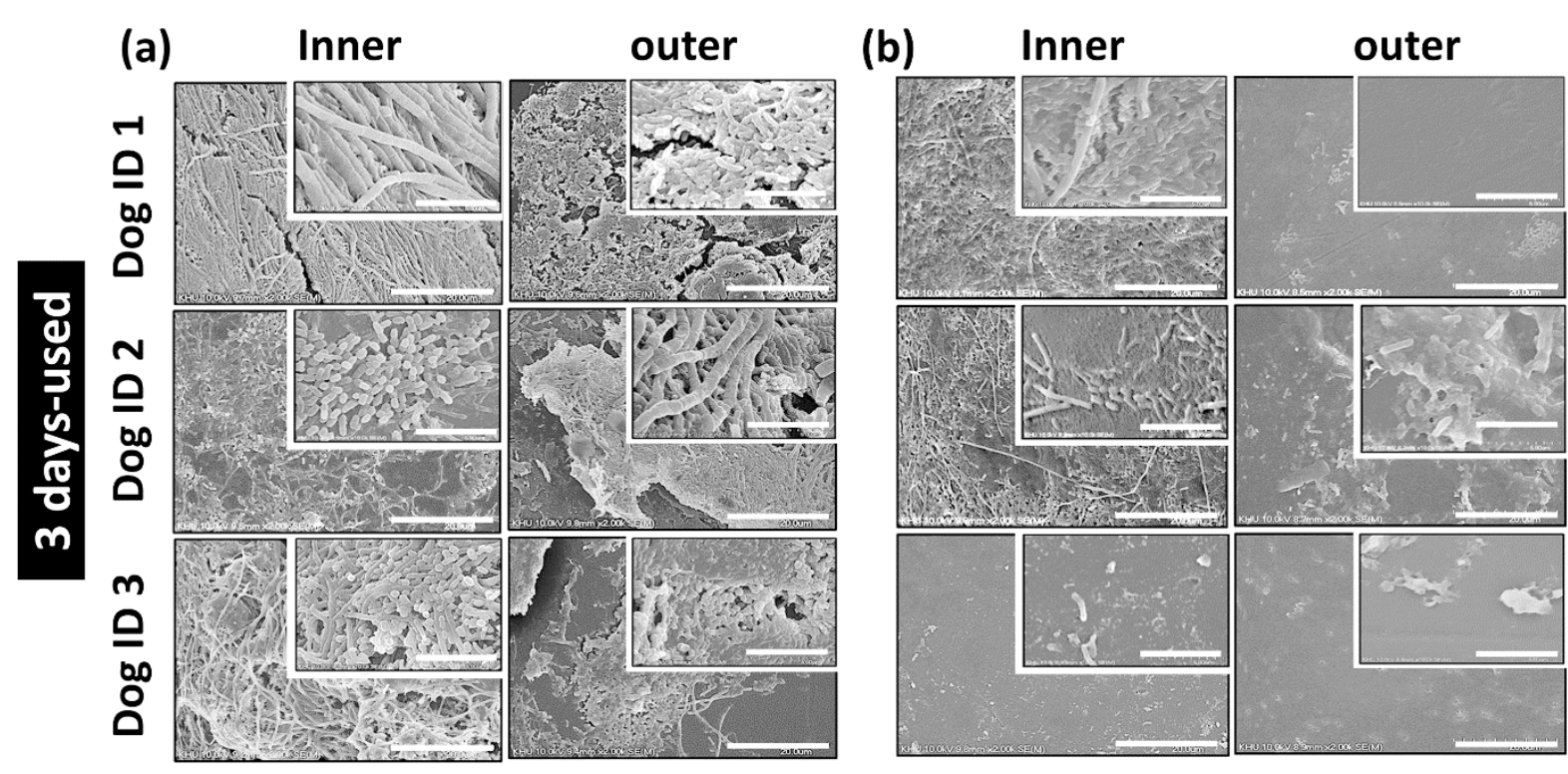

(c)

(d)
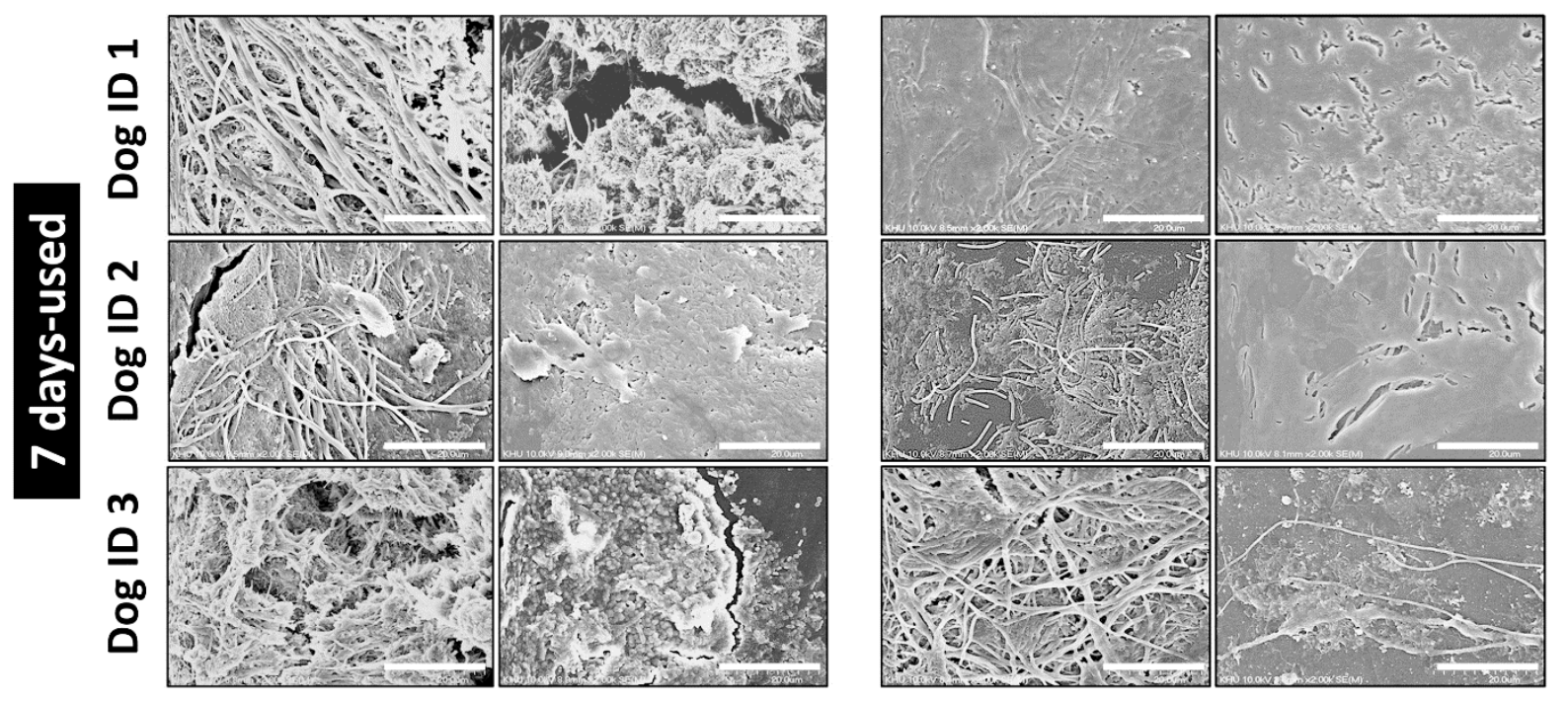

Figure S14. SEM images of carnie oral bacterial biofilm. Biofilm on the 3 or 7 days-used (a,c)

CTRL and (b,d) 50MPC/50SBMA. In each row (top, middle, and bottom), biofilms were obtained from the same beagle dog. The scale bar of the original and inserted SEM images individually indicates $20 \mu \mathrm{m}$ and $5.0 \mu \mathrm{m}$. 


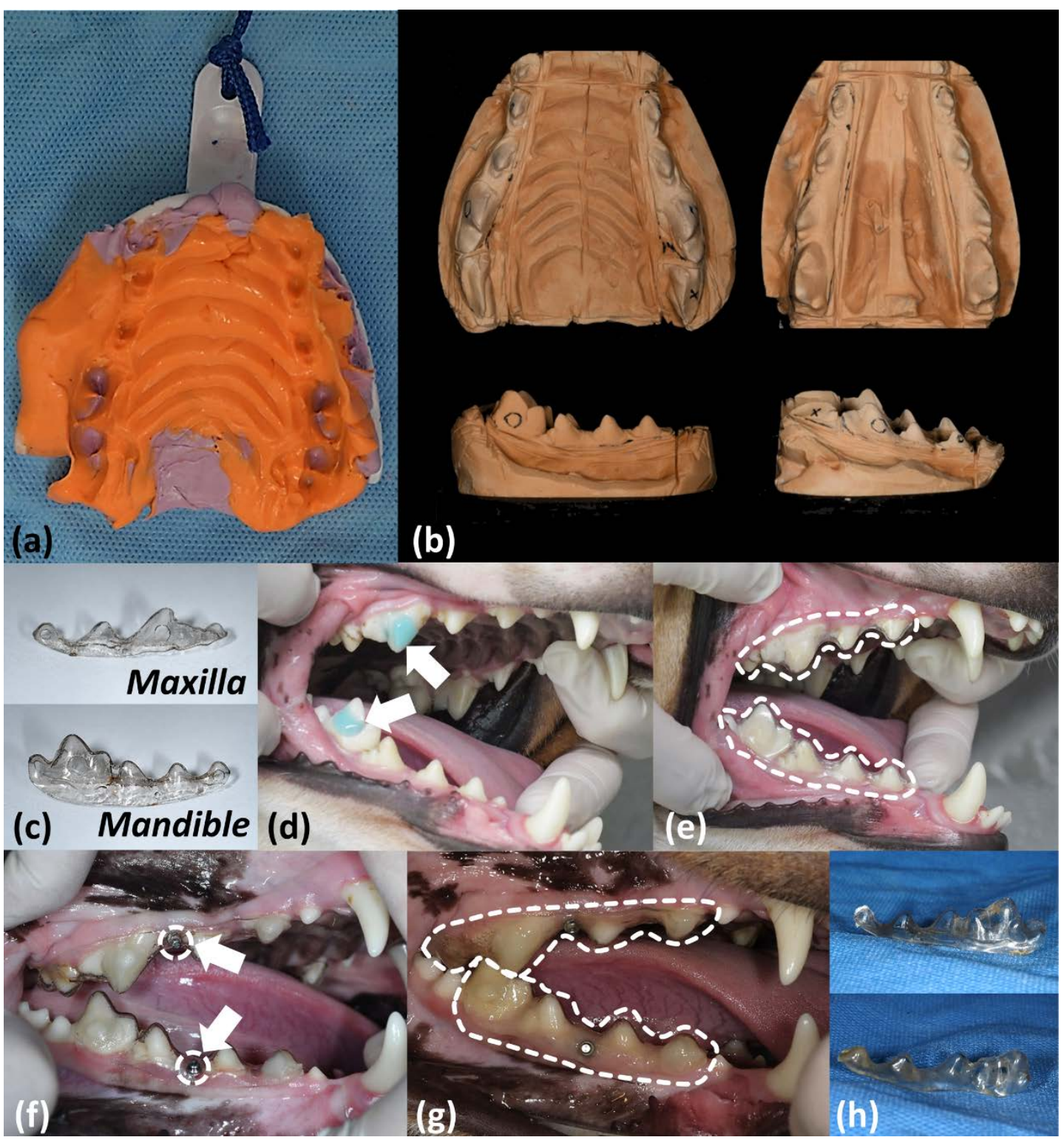

Figure S15. Procedures of in vivo study. (a) Impression with vinyl polysiloxane elastomeric silicone impression materials. (b) Dental casts of maxillary and mandibular arches. (c) Vacuum formed retainers (VFRs) with 50MPC/50SBMA on the maxilla (upper) and the mandible (lower). (d) Etching on the first molar using 37\% phosphoric acid after oral prophylaxis. (e) Delivery of VFRs and additional resin bonding. (f) Mini-implant installation for retention. (g) Intraoral photo after 7 days. (h) Removal of VFRs after 7 days. 
(a)

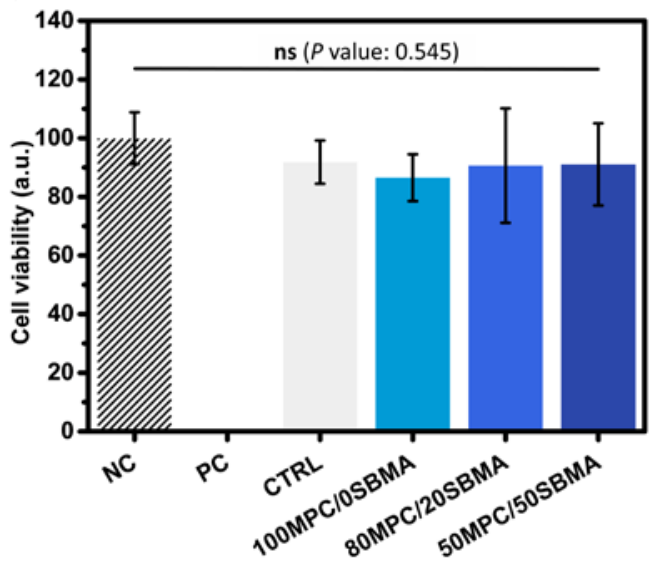

(b)
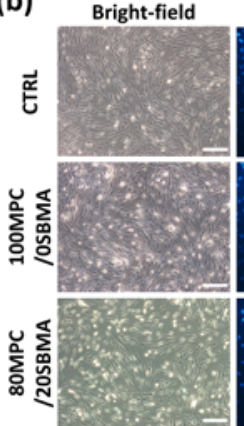

空蒡
DAPI (Nucleus) CMFDA (Cytoplasm)
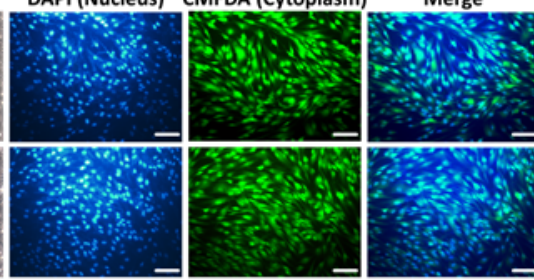

singes

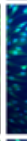

ह
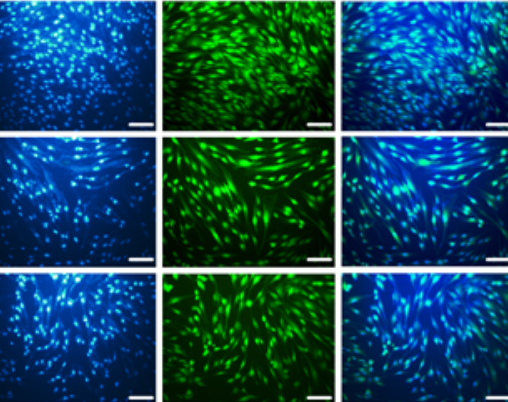

$\therefore$ Want $\because 2$

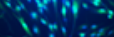

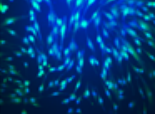

Figure S16. Cytotoxicity of synthesized ZPs. (a) Cytotoxicity assay via CCK assay, (b) Brightfield microscope, and counter-staining image. Scale bar indicates $200 \mu \mathrm{m}$. Human dermal fibroblast was exploited as the model cell.

To overcome the problematic resistance of biofilms, antibiotics or bactericidal materials are often used. However, the high dose of drugs is accompanied by cytotoxicity. ${ }^{11} 17$ Notably, our ZPs had no adverse effect on the metabolic activity of the model cells, human dermal fibroblast (Figure S16a). We also verified that our polymers were not cytotoxic because they did not affect innercell morphology, including the nucleus and cytoplasm (Figure S16b). Thus, 50MPC/50SBMA is a promising material for antifouling applications for clinical medical devices, showing superior inhibition of biofouling without cytotoxicity. 
Table S1. Characterization of ZPs: $\Gamma, \sigma$, and $D$

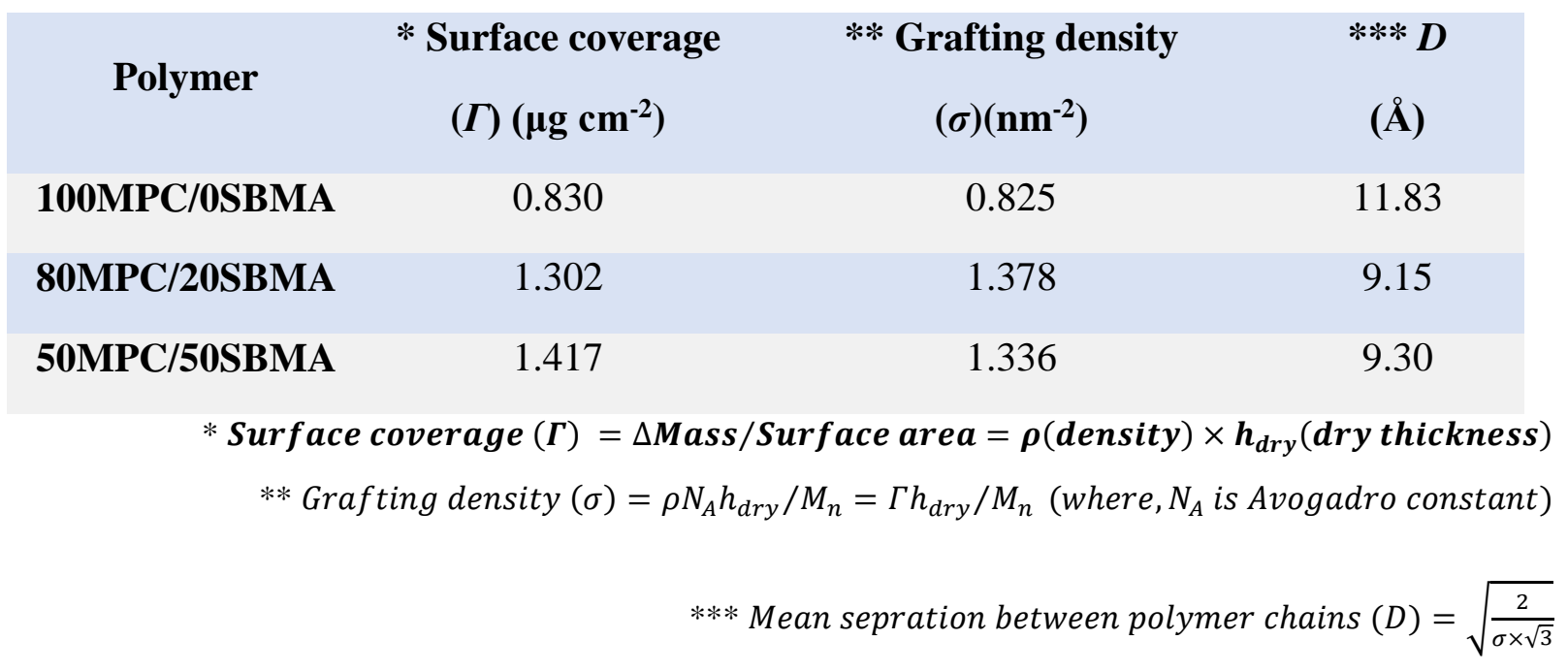


Table S2. Surface zeta-potential of ZPs

\begin{tabular}{|cc|}
\hline Polymer & $*$ Zeta-potential \\
\hline 100MPC/0SBMA & $2.16 \pm 0.354 \mathrm{mV}$ \\
\hline 80MPC/20SBMA & $1.76 \pm 0.396 \mathrm{mV}$ \\
\hline 50MPC/50SBMA & $0.74 \pm 0.516 \mathrm{mV}$ \\
& * The surface zeta potential was measured at neutral media.
\end{tabular}


Table S3. Quantification of RDF within the tail region

\begin{tabular}{cccc|} 
& 100MPC/0SBMA & 50MPC/50SBMA & 0MPC/100SBMA \\
Peak Intensity of tail & 3.59 & 3.80 & 4.67 \\
R $_{\text {tail }}$ & $6.14 \AA$ & $6.38 \AA$ & $6.38 \AA$
\end{tabular}


Table S4. Bacterial genus-specific primers used in PCR.

\begin{tabular}{|c|c|c|c|}
\hline Genus & Forward primer $\left(5^{\prime}-3^{\prime}\right)$ & Reverse primer $\left(5^{\prime}-3^{\prime}\right)$ & $\begin{array}{l}\text { Amplico } \\
\text { n (bp) }\end{array}$ \\
\hline Neisseria & $\begin{array}{l}\text { CGGGTTGTAAAGGACTTTT } \\
\text { G }\end{array}$ & $\begin{array}{l}\text { AGTTAGCCGGTGCTTATTC } \\
\mathrm{T}\end{array}$ & 135 \\
\hline Actinomyces & $\begin{array}{l}\text { GCGAAGAACCTTACCAAG } \\
\text { GC }\end{array}$ & $\begin{array}{l}\text { TGACGACAACCATGCACC } \\
\text { AC }\end{array}$ & 142 \\
\hline $\begin{array}{l}\text { Porphyromon } \\
\text { as }\end{array}$ & $\begin{array}{l}\text { CAGCCAAGTCGCGTGAAG } \\
\text { GA }\end{array}$ & $\begin{array}{l}\text { CTGGCACGGAGTTAGCCG } \\
\text { AT }\end{array}$ & 172 \\
\hline
\end{tabular}

Concerning human dental plaque formation, aerobic bacteria (such as Streptococcus, Neisseria, and Rothia) are the main initial colonizers of tooth surfaces. As plaque matures, the proportions of anaerobic genera (such as Actinomyces, Corynebacterium, and Fusobacterium) increase gradually. ${ }^{18}$ Compared with human oral microbiota, canine oral microbiota have been relatively less studied, but recent 16S rDNA pyrosequencing studies have identified over ca. 300 operational taxonomic units from the oral cavities of dogs. ${ }^{19} 20$ The bacterial community constituting canine plaques is widely different from humans. For example, Streptococcus Mutans is a vital virulence factor related to the etiology and pathogenesis of dental caries in human; however, Streptococcus species existed only very low level in dogs. However, some taxa are shared. For instance, many initial colonizers are Neisseria species, and later colonizers include species of Actinomyces and Porphyromonas. ${ }^{19}$ Thus, we chose Neisseria, Actinomyces, and Porphyromonas as the model bacterial genera to investigate the antifouling mechanism of 50MPC/50SBMA.; thus, Streptococcus Mutans was not model bacterial geneus.

In detail, Neisseria, Actinomyces, and Porphyromonas are significant pathogens. (i) Neisseria, Actinomyces, and Porphyromonas are classified as typical colonizers. Previous in vitro assays of biofilm formation identified the five primary colonizer species, three of which belonged to the 
genus Neisseria. ${ }^{19}$ In addition, Actinomyces and Porphyromonas are late-stage colonizers, interacting with Neisseria or Stenotrophomonas species before binding to the enamel. (ii) Actinomyces, Neisseria, and Porphyromonas are positively associated with gingivitis or periodontitis, and Porphyromonas is regarded as an analog of $P$. gingivalis. Thus, Actinomyces may play the same role in canine periodontitis that $P$. gingivalis plays in human periodontitis. ${ }^{21}$ Actinomyces and Neisseria are also considered as one of the components of human and animal gingival margin plaque. 


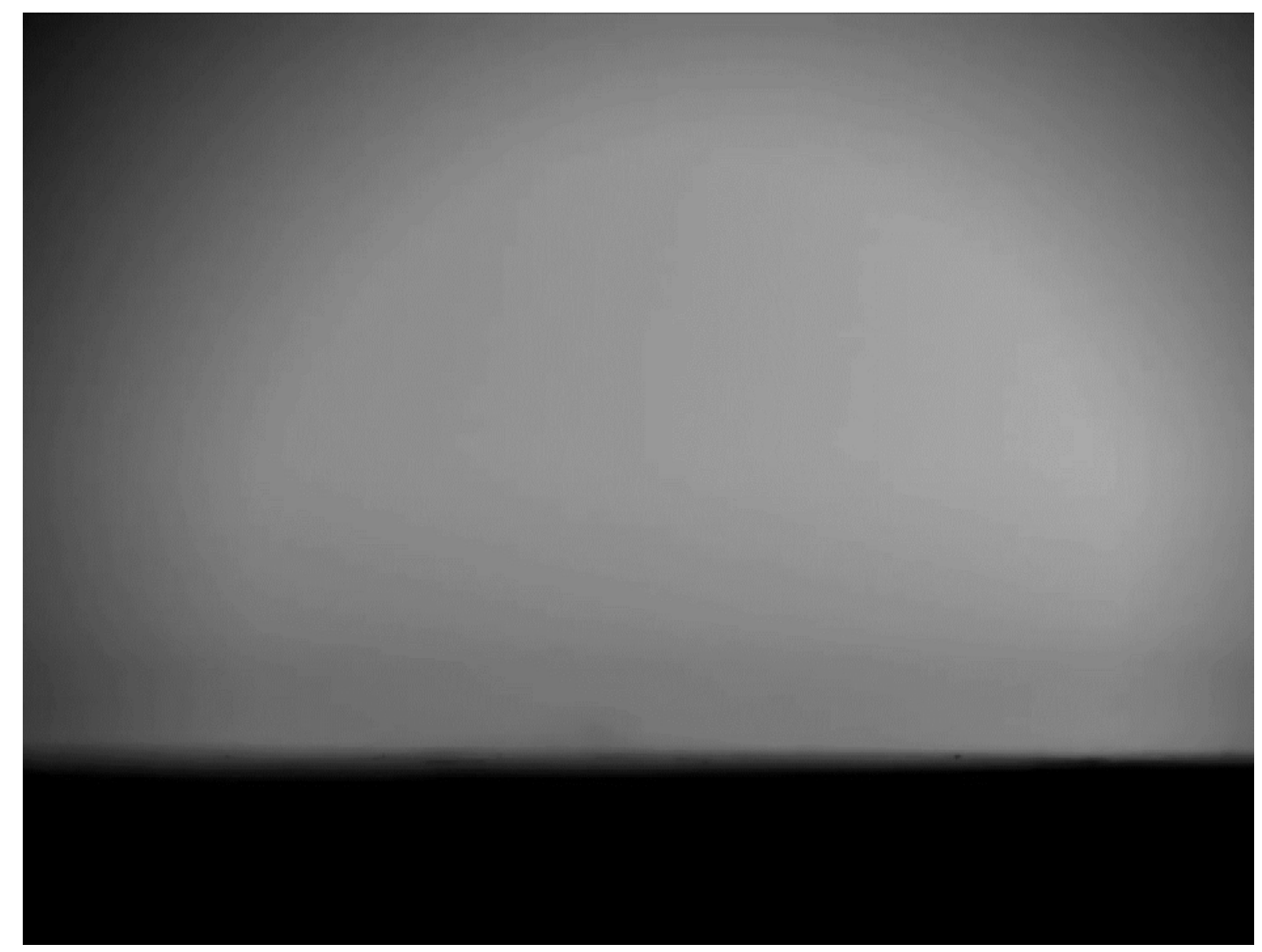

Movie S1. Super-hydrophilicity of 100MPC/0SBMA 


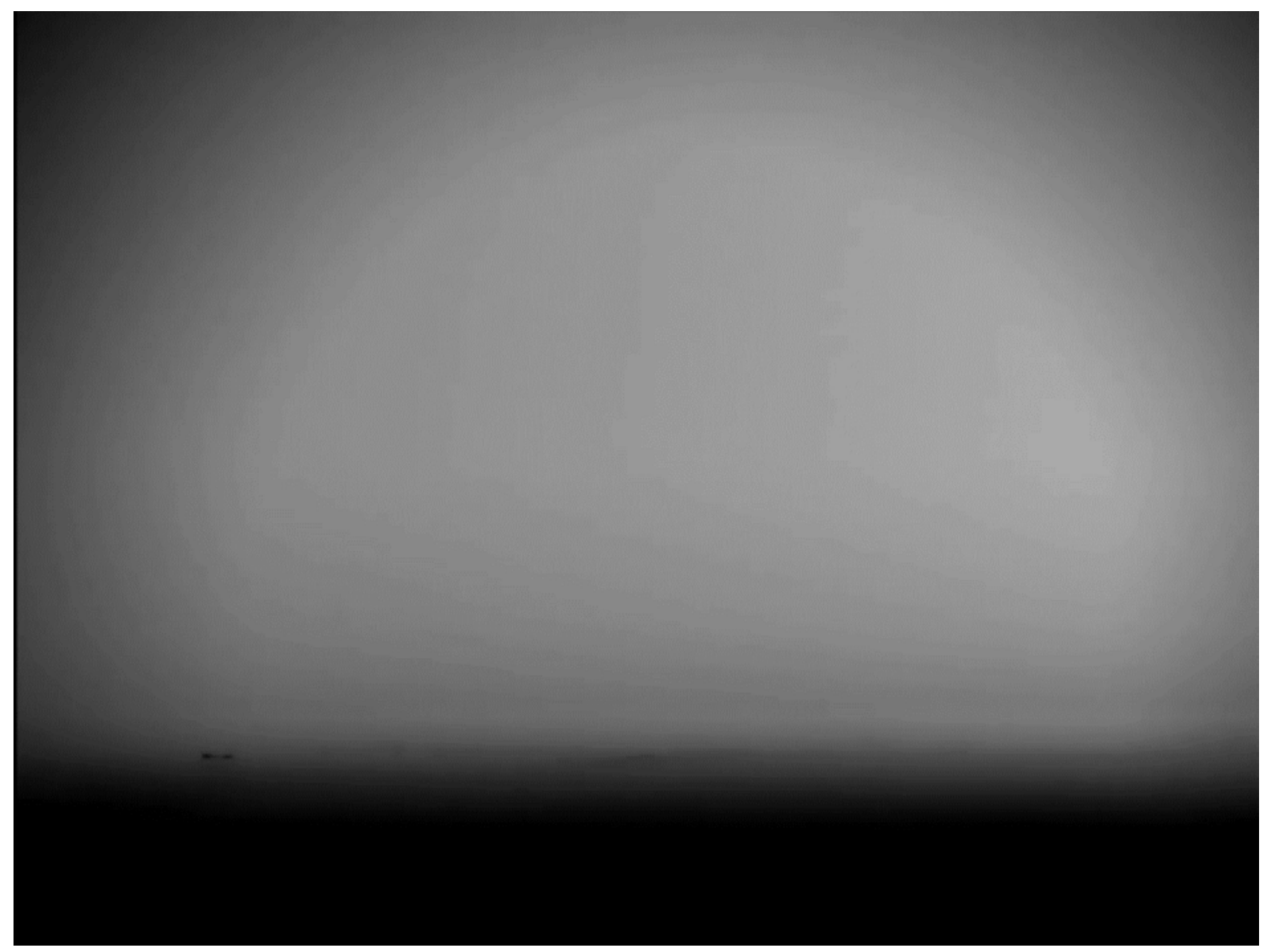

Movie S2. Super-hydrophilicity of 80MPC/20SBMA 


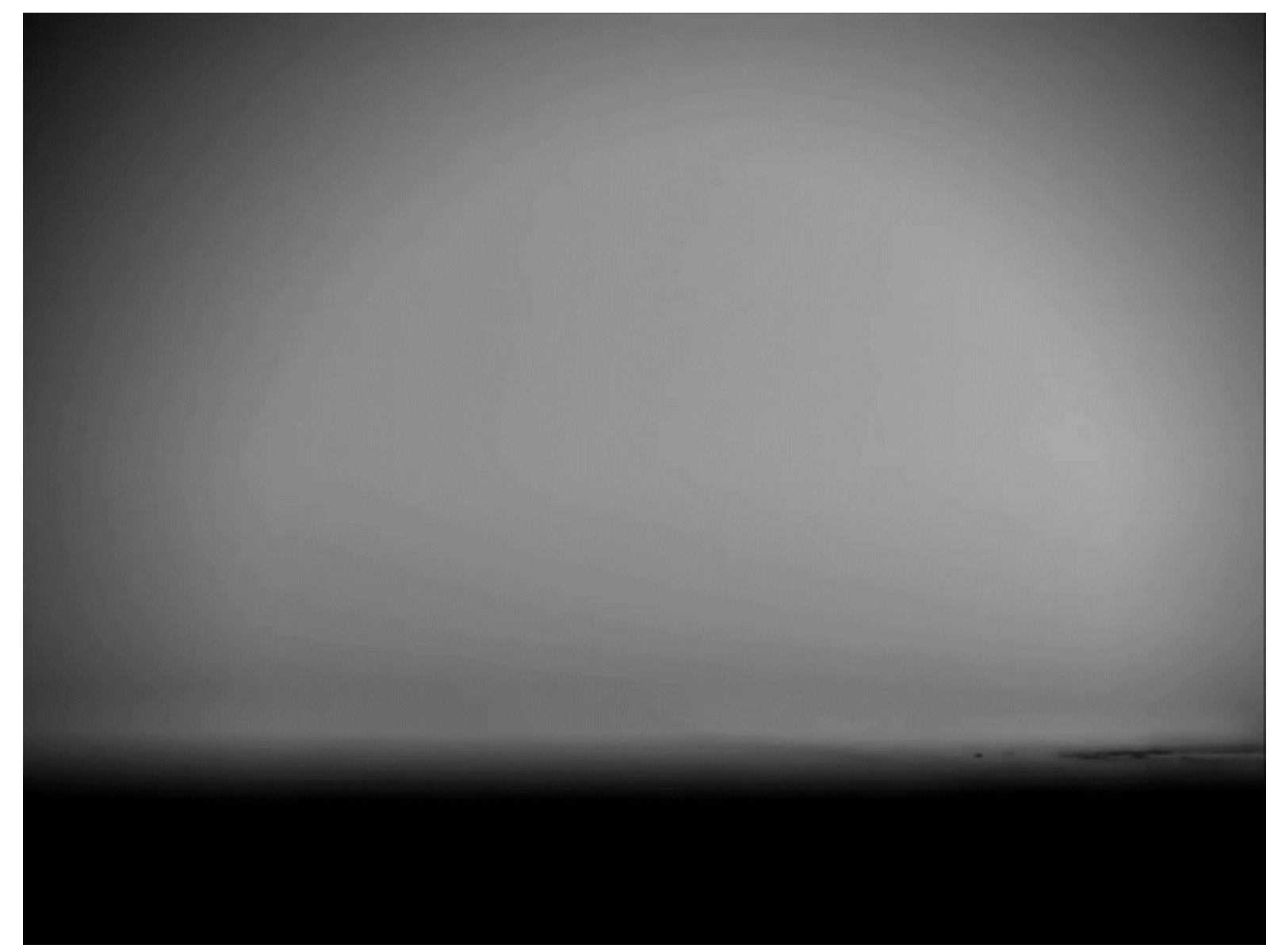

Movie S3. Super-hydrophilicity of 50MPC/50SBMA 


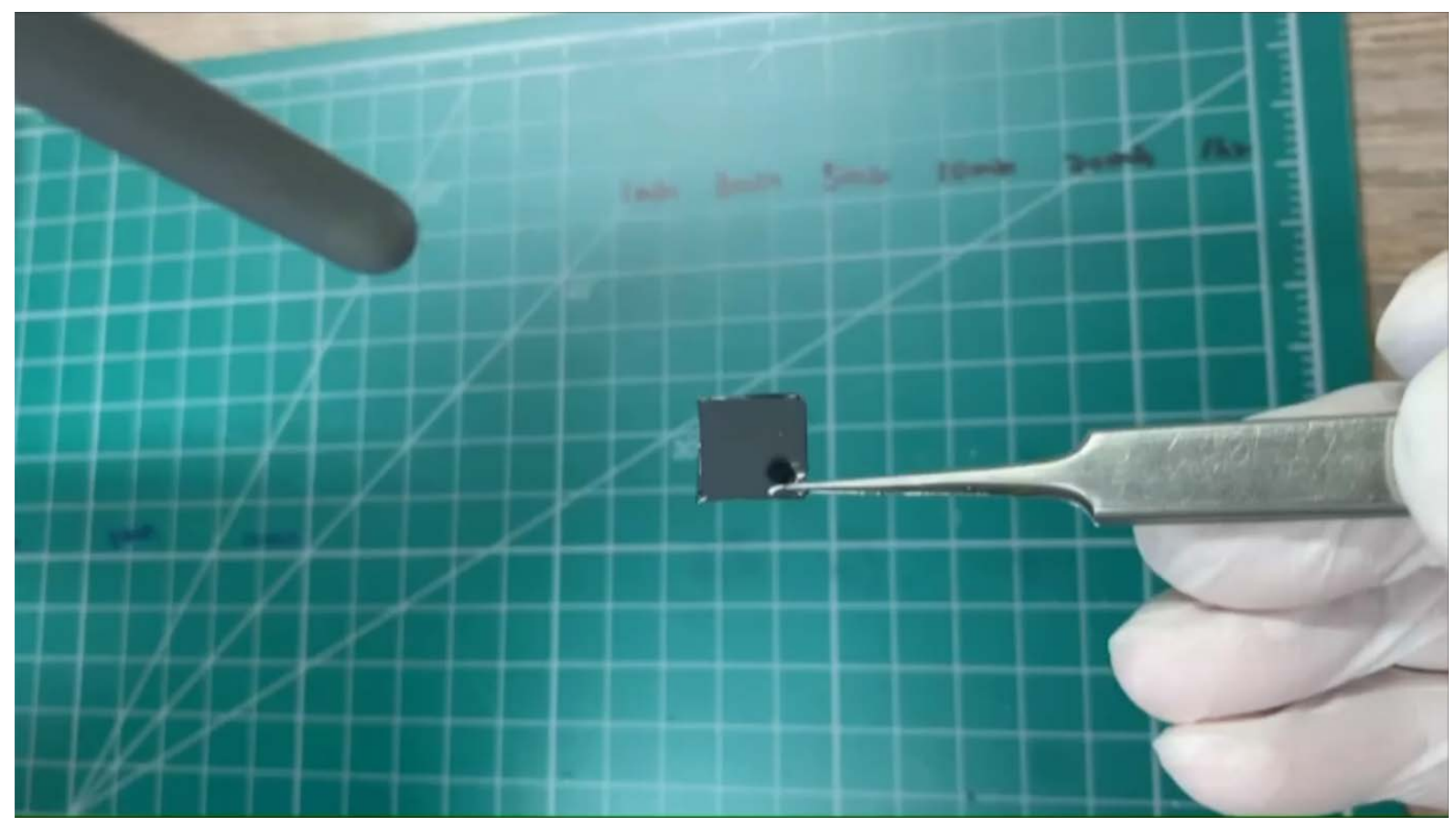

Movie S4. Non-gel like structure of representative zwitterionic random copolymer, 50MPC/50MPC. The typical blower was used to apply shear stress. 


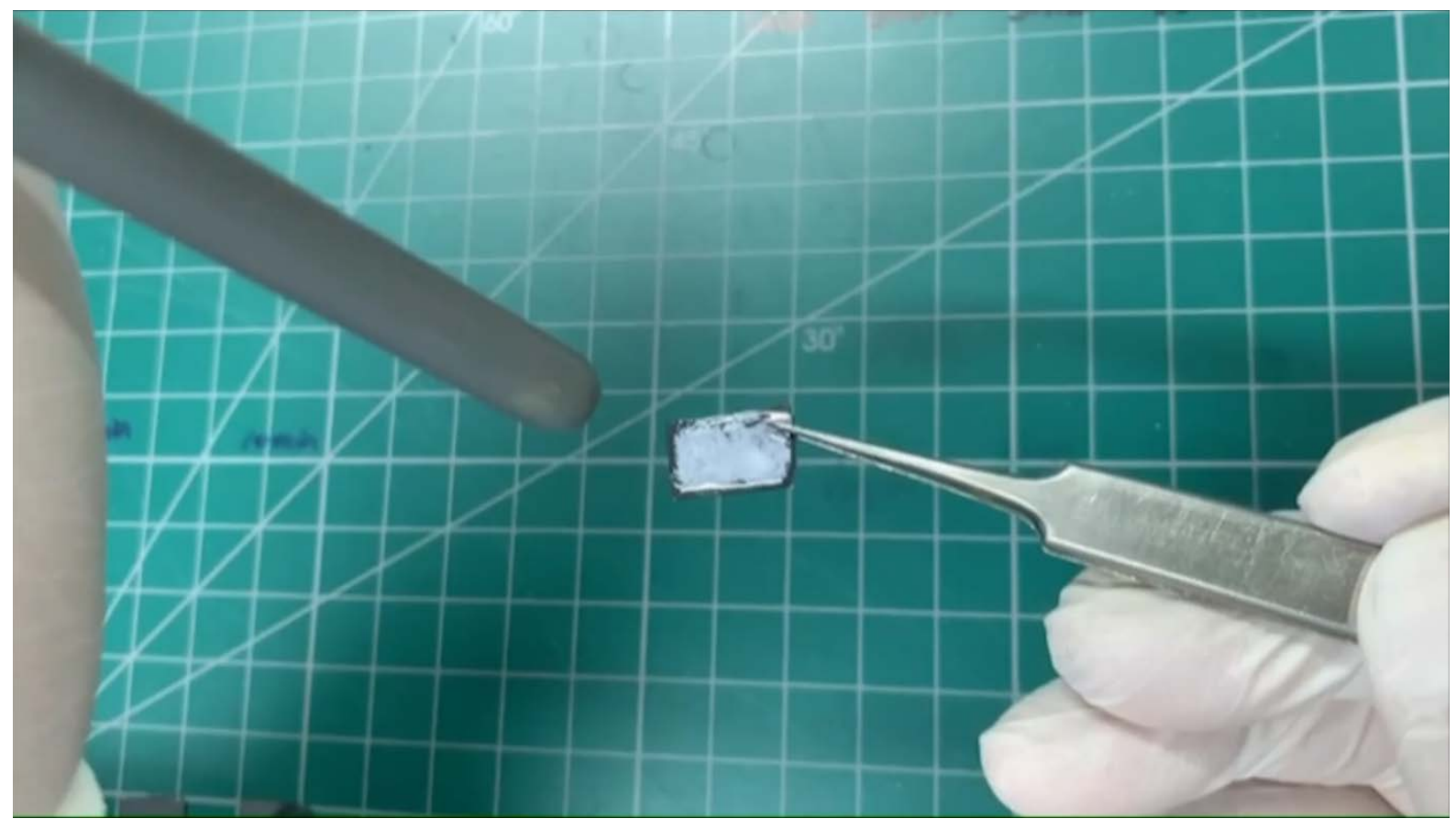

Movie S5. Gel like structure of 0MPC/100SBMA. The typical blower was used to apply shear stress. 


\section{REFERENCES for Figure 5c (Listed from the left symbol)}

1. Chen, H.; Zhang, M.; Yang, J.; Zhao, C.; Hu, R.; Chen, Q.; Chang, Y.; Zheng, J., Synthesis and Characterization of Antifouling Poly (N-Acryloylaminoethoxyethanol) with Ultralow Protein Adsorption and Cell Attachment. Langmuir 2014, 30, 10398-10409.

2. Liu, Y.; Nevanen, T. K.; Paananen, A.; Kempe, K.; Wilson, P.; Johansson, L.-S.; Joensuu, J. J.; Linder, M. B.; Haddleton, D. M.; Milani, R., Self-Assembling Protein-Polymer Bioconjugates for Surfaces with Antifouling Features and Low Nonspecific Binding. ACS Appl. Mater. Interfaces 2018, 11, 3599-3608.

3. Xia, Y.; Adibnia, V.; Shan, C.; Huang, R.; Qi, W.; He, Z.; Xie, G.; Olszewski, M.; De Crescenzo, G.; Matyjaszewski, K., Synergy between Zwitterionic Polymers and Hyaluronic Acid Enhances Antifouling Performance. Langmuir 2019, 35, 15535-15542.

4. Li, G.; Cheng, G.; Xue, H.; Chen, S.; Zhang, F.; Jiang, S., Ultra Low Fouling Zwitterionic Polymers with a Biomimetic Adhesive Group. Biomaterials 2008, 29, 4592-4597.

5. Li, L.; Yan, B.; Zhang, L.; Tian, Y.; Zeng, H., Mussel-Inspired Antifouling Coatings Bearing Polymer Loops. ChemComm 2015, 51, 15780-15783.

6. Chen, H.; Yang, J.; Xiao, S.; Hu, R.; Bhaway, S. M.; Vogt, B. D.; Zhang, M.; Chen, Q.; Ma, J.; Chang, Y., Salt-Responsive Polyzwitterionic Materials for Surface Regeneration between Switchable Fouling and Antifouling Properties. Acta Biomater. 2016, 40, 62-69.

7. Rodriguez-Emmenegger, C.; Houska, M.; Alles, A. B.; Brynda, E., Surfaces Resistant to Fouling from Biological Fluids: Towards Bioactive Surfaces for Real Applications. Macromol Biosci 2012, 12, 1413-1422. 
8. Goh, S.; Luan, Y.; Wang, X.; Du, H.; Chau, C.; Schellhorn, H.; Brash, J.; Chen, H.; Fang, Q., Polydopamine-Polyethylene Glycol-Albumin Antifouling Coatings on Multiple Substrates. $J$ Mater Chem B 2018, 6, 940-949.

9. Qi, H.; Zheng, W.; Zhou, X.; Zhang, C.; Zhang, L., A Mussel-Inspired Chimeric Protein as a Novel Facile Antifouling Coating. ChemComm 2018, 54, 11328-11331.

10. Hecker, M.; Ting, M. S. H.; Malmström, J., Simple Coatings to Render Polystyrene Protein Resistant. Coatings 2018, 8, 55.

11. Goor, O. J.; Brouns, J. E.; Dankers, P. Y., Introduction of Anti-Fouling Coatings at the Surface of Supramolecular Elastomeric Materials via Post-Modification of Reactive Supramolecular Additives. Polym. Chem. 2017, 8, 5228-5238.

12. Zhao, X.; Xuan, H.; He, C., Enhanced Separation and Antifouling Properties of Pvdf Ultrafiltration Membranes with Surface Covalent Self-Assembly of Polyethylene Glycol. RSC Adv. 2015, 5, 81115-81122.

13. Zhao, Y.-F.; Zhu, L.-P.; Yi, Z.; Zhu, B.-K.; Xu, Y.-Y., Zwitterionic Hydrogel Thin Films as Antifouling Surface Layers of Polyethersulfone Ultrafiltration Membranes Anchored via Reactive Copolymer Additive. J. Membr. Sci. 2014, 470, 148-158.

14. Kim, S.-E.; Zhang, C.; Advincula, A. A.; Baer, E.; Pokorski, J. K., Protein and Bacterial Antifouling Behavior of Melt-Coextruded Nanofiber Mats. ACS Appl. Mater. Interfaces 2016, 8, 8928-8938.

15. Shao, L.; Wang, Z. X.; Zhang, Y. L.; Jiang, Z. X.; Liu, Y. Y., A Facile Strategy to Enhance Pvdf Ultrafiltration Membrane Performance via Self-Polymerized Polydopamine Followed by Hydrolysis of Ammonium Fluotitanate. J. Membr. Sci. 2014, 461, 10-21. 
16. Ghaleh, H.; Jalili, K.; Maher, B. M.; Rahbarghazi, R.; Mehrjoo, M.; Bonakdar, S.; Abbasi, F., Biomimetic Antifouling Pdms Surface Developed via Well-Defined Polymer Brushes for Cardiovascular Applications. Eur. Polym. J. 2018, 106, 305-317.

17. Wu, T.; Zhou, B.; Zhu, T.; Shi, J.; Xu, Z.; Hu, C.; Wang, J., Facile and Low-Cost Approach towards a Pvdf Ultrafiltration Membrane with Enhanced Hydrophilicity and Antifouling Performance via Graphene Oxide/Water-Bath Coagulation. RSC Adv. 2015, 5, 78807889.

18. Yu, L.; Zhang, Y.; Zhang, B.; Liu, J.; Zhang, H.; Song, C., Preparation and Characterization of Hpei-Go/Pes Ultrafiltration Membrane with Antifouling and Antibacterial Properties. J. Membr. Sci. 2013, 447, 452-462.

19. Shen, X.; Liu, P.; Xia, S.; Liu, J.; Wang, R.; Zhao, H.; Liu, Q.; Xu, J.; Wang, F., AntiFouling and Anti-Bacterial Modification of Poly (Vinylidene Fluoride) Membrane by Blending with the Capsaicin-Based Copolymer. Polymers 2019, 11, 323.

20. Shen, Z.; Chen, W.; Xu, H.; Yang, W.; Kong, Q.; Wang, A.; Ding, M.; Shang, J., Fabrication of a Novel Antifouling Polysulfone Membrane with in Situ Embedment of Mxene Nanosheets. INT J ENV RES PUB HE 2019, 16, 4659. 


\section{REFERENCES for Supporting Information}

1. Takahashi, H.; Nakayama, M.; Yamato, M.; Okano, T., Controlled Chain Length and Graft

Density of Thermoresponsive Polymer Brushes for Optimizing Cell Sheet Harvest.

Biomacromolecules 2010, 11, 1991-1999.

2. Asai, M.; Zhao, D.; Kumar, S. K., Role of Grafting Mechanism on the Polymer Coverage and Self-Assembly of Hairy Nanoparticles. ACS Nano 2017, 11, 7028-7035.

3. Yah, W. O.; Xu, H.; Soejima, H.; Ma, W.; Lvov, Y.; Takahara, A., Biomimetic Dopamine Derivative for Selective Polymer Modification of Halloysite Nanotube Lumen. J. Am. Chem. Soc. 2012, 134, 12134-12137.

4. Akram Bhuiyan, M. S.; Roland, J. D.; Liu, B.; Reaume, M.; Zhang, Z.; Kelley, J. D.; Lee, B. P., In Situ Deactivation of Catechol-Containing Adhesive Using Electrochemistry. J. Am. Chem. Soc. 2020, 142, 4631-4638.

5. Kang, C.; Crockett, R. M.; Spencer, N. D., Molecular-Weight Determination of Polymer Brushes Generated by Si-Atrp on Flat Surfaces. Macromolecules 2014, 47, 269-275.

6. Moh, L. C.; Losego, M. D.; Braun, P. V., Solvent Quality Effects on Scaling Behavior of Poly (Methyl Methacrylate) Brushes in the Moderate-and High-Density Regimes. Langmuir 2011, 27, 3698-3702.

7. Mathis, C. H.; Kang, C.; Ramakrishna, S. N.; Isa, L.; Spencer, N. D., Indenting Polymer Brushes of Varying Grafting Density in a Viscous Fluid: A Gradient Approach to Understanding Fluid Confinement. Polymer 2019, 169, 115-123.

8. Mayo, F. R.; Lewis, F. M., Copolymerization. I. A Basis for Comparing the Behavior of Monomers in Copolymerization; the Copolymerization of Styrene and Methyl Methacrylate. $J$. Am. Chem. Soc. 1944, 66, 1594-1601. 
9. Lin, T.-P.; Chang, A. B.; Chen, H.-Y.; Liberman-Martin, A. L.; Bates, C. M.; Voegtle, M. J.; Bauer, C. A.; Grubbs, R. H., Control of Grafting Density and Distribution in Graft Polymers by Living Ring-Opening Metathesis Copolymerization. J. Am. Chem. Soc. 2017, 139, 38963903.

10. Xiang, T.; Zhang, L.-S.; Wang, R.; Xia, Y.; Su, B.-H.; Zhao, C.-S., Blood Compatibility Comparison for Polysulfone Membranes Modified by Grafting Block and Random Zwitterionic Copolymers via Surface-Initiated Atrp. J. Colloid Interface Sci. 2014, 432, 47-56.

11. Choi, W.; Jin, J.; Park, S.; Kim, J.-Y.; Lee, M.-J.; Sun, H.; Kwon, J.-S.; Lee, H.; Choi, S.-H.; Hong, J., Quantitative Interpretation of Hydration Dynamics Enabled the Fabrication of a Zwitterionic Antifouling Surface. ACS Appl. Mater. Interfaces 2020, 12, 7951-7965.

12. Raman Spectroscopic Study of the Effects of Dissolved Nacl on Water Structure. Vib Spectrosc 2012, 62, 110-114.

13. Auer, B.; Kumar, R.; Schmidt, J.; Skinner, J., Hydrogen Bonding and Raman, Ir, and 2d-Ir Spectroscopy of Dilute Hod in Liquid D2o. Proc. Natl. Acad. Sci. U.S.A. 2007, 104, 1421514220.

14. Perakis, F.; De Marco, L.; Shalit, A.; Tang, F.; Kann, Z. R.; Kühne, T. D.; Torre, R.; Bonn, M.; Nagata, Y., Vibrational Spectroscopy and Dynamics of Water. Chem. Rev. 2016, 116, 7590-7607.

15. Oh, J. K.; Yegin, Y.; Yang, F.; Zhang, M.; Li, J.; Huang, S.; Verkhoturov, S. V.; Schweikert, E. A.; Perez-Lewis, K.; Scholar, E. A., The Influence of Surface Chemistry on the Kinetics and Thermodynamics of Bacterial Adhesion. Sci. Rep. 2018, 8, 1-13. 
16. Kobayashi, M.; Terayama, Y.; Kikuchi, M.; Takahara, A., Chain Dimensions and Surface Characterization of Superhydrophilic Polymer Brushes with Zwitterion Side Groups. Soft Matter 2013, 9, 5138-5148.

17. Liao, C.; Li, Y.; Tjong, S. C., Bactericidal and Cytotoxic Properties of Silver Nanoparticles. Int. J. Mol. Sci. 2019, 20, 449.

18. Ritz, H., Microbial Population Shifts in Developing Human Dental Plaque. Arch. Oral Biol. 1967, 12, 1561-1568.

19. Holcombe, L. J.; Patel, N.; Colyer, A.; Deusch, O.; O’Flynn, C.; Harris, S., Early Canine Plaque Biofilms: Characterization of Key Bacterial Interactions Involved in Initial Colonization of Enamel. PLoS One 2014, 9, e113744.

20. Ruparell, A.; Inui, T.; Staunton, R.; Wallis, C.; Deusch, O.; Holcombe, L. J., The Canine Oral Microbiome: Variation in Bacterial Populations across Different Niches. BMC Microbiol. 2020, 20, 1-13.

21. Takada, K.; Hirasawa, M., Expression of Trypsin-Like Activity by the Genera Corynebacterium and Actinomyces in Canine Periodontitis. Int. J. Med. Microbiol. 2000, 49, 621-625. 